\title{
Competition with asymmetric switching costs
}

\author{
S. Infante \\ N. Figueroa \\ R. Fischer \\ U. de Chile \\ U. de Chile ${ }^{1}$
}

November 1, 2007

${ }^{1}$ N. Figueroa and R. Fischer acknowledge the support of the Complex Engineering Systems Institute. 


\begin{abstract}
We analyze the effects of asymmetric switching costs on two identical firms that produce an homogeneous good and compete in prices. Both firms inherit a fraction of the market which is "locked-in" by the switching costs. When switching costs are low, firms face a tradeoff between charging a high price to their locked in customers, or pricing aggressively in order to attract the rival's market share. We characterize the (pure and mixed) equilibrium strategies and the associated payoffs for any pair of switching costs in the unit square.
\end{abstract}




\section{Introduction}

In various markets, consumers are constantly faced with switching costs when they try to change their purchasing behavior. These switching costs often reduce competition among firms, giving companies a certain degree of monopoly power over their customer base. Clear examples of this can be seen in several markets, for example in the information technology sector where the choice of a certain hardware conditions the choice and availability of other types of products, creating additional costs to change from one company to another; or in the cellular phone service industry where often firms demand the cellular phone be returned when changing service provider. These costs are internalize by consumers. This fact may allow firms to raise prices to their initial market share without fearing the loss of their market participation.

In this paper we study the firms' behavior and payoffs when consumers are faced with asymmetric switching costs. The model analyzed in this paper consists of two identical firms that engage in a one time price competition and trying to maximize expected payoff. We start with an initial split of the market between firms and consider fixed switching costs that consumers must directly pay to the firm that is being left. Since our interest lies in characterizing the firms pricing behavior, knowing the switching costs of their customer base and that of their rivals, we shall exclude other factors such as the quality or quantity that might alter the firms' or consumers' decisions.

We will analyze two cases, one in which a firm has the entire market and another when the market is split in two equal parts among firms. In many cases, the switching costs will be so high that consumers are going to be completely locked-in to one firm, giving that company the ability to charge the monopoly price to its consumers. In other cases, the magnitude of the switching cost shall allow firms to price more aggressively to try to poach their rivals customer base. We will characterize the firms' payoffs under any possible pair of switching costs that consumers might face.

More specifically, we find that if both switching costs are above a certain threshold, it is not profitable for firms to poach their rivals consumers and thus both firms charge the maximum price possible, since consumers are completely locked in. This is the only region where a pure strategy equilibrium exists. When one of the firms switching costs is below the fore mentioned threshold but the sum of both switching costs is relatively high, we find a mixed strategy equilibrium in which only one firm has the possibility to poach his rival's consumers. This implies that only one of the firms strategy places a positive probability on relatively low prices in an attempt to capture the entire market. We call this equilibrium a single sided poaching equilibrium. The last type of mixed strategy equilibrium that we find is when both switching costs are relatively small or in a subset of the single sided poaching equilibrium, where both firms may poach the others market share. For this type of equilibrium both firms strategies place a positive probability on high prices to gain the most from their inherited market share and they also place a positive probability on low prices in an attempt to attract the other firms customers. We call these type of equilibrium double sided poaching equilibrium. 
Much work has been written in the switching cost literature. In [12] Klemperer reviews several models that incorporate switching costs with different characteristics. In the simplest model, he considers a duopoly that competes in two periods, where in the second period firms face an exogenous switching cost that is identical between firms and is so large that consumers are completely locked in to their initially chosen firm. In this context he manages to prove that first period competition is fierce enough to eliminate firms future margins. He also considers a model in which the goods offered by each firm are not perfect substitutes, giving leeway so that firms may extract a positive payoff from their consumers. Again, he assumes that the switching cost is elevated and that it is unfeasible for a consumer to change firms. Klemperer also studies the effects switching costs have when consumers change their preferences in different periods [11] or when consumers have idiosyncratic switching costs [10]. A common feature of this literature is that switching costs are so high that consumers do not have incentives to change firms. As a consequence consumers make their decisions only once, before they face any switching cost.

In [15] Shilony relaxes the assumption that consumers cannot change firm when they face switching costs. His paper considers $n$ firms that are separated across different neighborhoods and if consumers want to purchase from a seller that is "far away", they must pay a fixed transportation cost $c$. For small values of $c$ firms face a trade off between imposing a high price to satisfy their respective market share ("milk" their own customer base) or impose a low price to attract consumers from different neighborhoods ("poach" their competitors customer base). This trade off leads to a mixed strategy equilibrium. We extend this model to consider asymmetric switching costs among firms, which in some cases may give one firm an advantage over his rival. An important difference is that in our model the firms receive the switching cost that must be paid if a customer decides to switch, whereas in Shilony's model the cost is seen as a transportation cost and is wasted.

We see this paper as the characterization of the final period of a dynamic oligopoly model. In the first period of this model firms have two choices to make: what price to charge consumers and what incentive to offer to attract them ${ }^{1}$. This incentive is seen as a gift in the first period but it later turns into a switching cost since it must be returned in case a consumer would like to switch firms in the second period. This generates a potentially asymmetric situation in the final period, where we may have an asymmetric market distribution and where consumers must face different switching costs (depending on which provider they selected in the previous period).

The distribution of this paper is as follows. Section 2 gives a description of the model considered. In section 3 we characterize all of the possible resulting equilibria that can arise from this type of competition. We find the exact conditions necessary for firms to be able to fix the monopoly price without loosing their market share, and the resulting mixed strategy equilibrium when switching costs are relatively low and firms may poach their rivals consumers. In section 4 we give an intuitive explication of the

\footnotetext{
${ }^{1}$ For example, a new cellular phone offered by cellular phone service companies or an initial teaser rate on a home mortgages.
} 
equilibria characterize in the previous section, and finally in section 5 we conclude.

\section{The Model}

There are two firms $i$ and $j$ competing in price (denoted by $p_{i}$ and $p_{j}$ respectively) over one period. We assume that firm $i$ has inherited a market share $\mu_{i} \in\left\{0, \frac{1}{2}, 1\right\}$ ( $\mu_{j}$ denotes $j$ 's initial market share), which is the proportion of the market inherited by firm $i^{2}$. If a consumer is inherited by firm $i$ she must pay a switching $\operatorname{cost} B_{i}$ to that firm in order to change providers. We assume that the firms are identical, with their marginal costs normalized to zero.

Note that in this model we do not assume that it is too costly for consumers to switch; consumers may choose to do so, depending on the price difference between firms. This means that for a consumer inherited by $i$ to switch to firm $j$ the price difference must be greater than the switching $\operatorname{cost} B_{i}$, in other words $p_{i}>p_{j}+B_{i}$. We assume that firms cannot differentiate their prices, therefore all consumers face the same prices no matter which firm they are initially attached to; and that the consumers' reservation price is 1 , hence $p_{i}, p_{j} \in[0,1]$ and $B_{i}, B_{j} \in[0,1]$. This gives us the following demand function for firm $i$,

$$
D_{i}\left(p_{i}, p_{j}\right)= \begin{cases}0 & \text { if } p_{i}>p_{j}+B_{i} \\ \mu_{i} & \text { if } p_{j}-B_{j} \leq p_{i} \leq p_{j}+B_{i} \\ 1 & \text { if } p_{i}<p_{j}-B_{j}\end{cases}
$$

And firm $i$ 's payoff takes on the following form,

$$
\pi_{i}\left(p_{i}, p_{j}\right)= \begin{cases}\mu_{i} B_{i} & \text { if } p_{i}>p_{j}+B_{i} \\ \mu_{i} p_{i} & \text { if } p_{j}-B_{j} \leq p_{i} \leq p_{j}+B_{i} \\ p_{i} & \text { if } p_{i}<p_{j}-B_{j}\end{cases}
$$

From this payoff function we can see that firms are torn between two choices, they can either charge a high price to extract the most from their "locked-in" consumers ${ }^{3}$ or they can charge a lower price so as to attract their rival's market share. For some levels of $B_{i}, B_{j}$, this tradeoff generates mixed strategy equilibria.

\footnotetext{
${ }^{2}$ This can be interpreted as the proportion of the market that has purchased from $i$ before.

${ }^{3}$ Here we use the term "locked-in" loosely, since consumers have the option of changing firms, but they face a switching cost in order to do so.
} 


\section{Characterizations of Equilibria}

In this section we shall characterize all of the possible equilibria that can arise in the game. They depend strongly on the magnitude of the switching costs that consumers must pay in order to switch firms. We fist study the case of a dominant firm that inherits the entire market $\left(\mu_{i} \in\{0,1\}\right)$. We find that there exists a pure strategy equilibrium where the dominant firm retains the whole market. We then consider the case in where both firms share the market in equal parts $\left(\mu_{i}=\frac{1}{2}\right)$ and find 5 types of equilibria. In the presence of relatively high switching costs we find a pure strategy Nash equilibrium where both firms charge a monopoly price and do not loose market share. For all other cases we prove that there is no pure strategy equilibria and characterize 4 different mixed strategy equilibria.

These 4 cases of mixed strategy equilibria can be separated in two types of strategies: 1 corresponds to a single sided poaching equilibrium and 3 correspond to a double sided poaching equilibrium. In the single sided poaching equilibrium we have that only one of the firms has the chance to poach his rivals consumers. Informally this means that both firms strategies place a positive probability on high prices to "milk" their consumers, but only one of the firms strategy places a positive probability on relatively low prices so as to capture the entire market. In the double sided poaching equilibrium both firms have the ability to poach their rivals consumers. For these types of equilibria both of the firms strategy support small prices to poach the other customer base and they also support high prices to "milk" their existing market share. This final type of equilibria is later divided in three sub cases, which depend on the magnitude of the firms' switching cost.

\subsection{Dominant Firm}

In this subsection we find a pure strategy equilibrium of the game when $j$ inherits the entire market.

Proposition 3.1. If $\mu_{i}=0$, the resulting equilibrium is

$$
p_{i}^{*}=0, p_{j}^{*}=B_{j}
$$

\section{Proof:}

With $\mu_{i}=0$ we have that firm $j$ inherits the entire market before the beginning of the game. Let us verify that $p_{i}^{*}=0, p_{j}^{*}=B_{j}$ constitutes an equilibrium. With these price levels, the firms' payoffs are,

$$
\pi_{i}=\mu_{i} p_{i}^{*}=0, \quad \pi_{j}=\left(1-\mu_{i}\right) p_{j}^{*}=B_{j}
$$

Given the consumer's demand function, if $j$ raises its price in $\epsilon>0$, all of its customers will be better off returning $j$ 's first period bond and purchasing from $i$. And if $j$ reduces its price by $\epsilon>0$, it will keep its market share but will receive a lower payoff because of the price reduction. Therefore, firm $j$ does not have an incentive to change its price. 
If firm $i$ decided to raise its price, the price difference between the two firms will be even smaller and consumers will have even fewer incentives to purchase from $i$, leaving $i$ with the same payoff as before the change in price. If firm $i$ reduces its price by $\epsilon>0$, it would sell to the entire market since consumers are better off returning the switching cost to $j$ and purchasing from $i$. But firm $i$ would receive a negative payoff since $\pi_{2 i}=-\epsilon<0$, so it has no incentives to lower its price. Therefore, since neither firm has incentives to modify its price, $p_{i}^{*}=0, p_{j}^{*}=B_{j}$ is an equilibrium.

This result is quite intuitive: if one firm has the entire market and consumers are tied by a switching cost $B$, then $B$ is the maximum price the dominant firm can charge to maintain its market share. In fact it is optimal for the firm to charge that price and keep the whole market.

\subsection{A Shared Market}

The characteristics of the equilibria are strongly linked to the size of the switching costs. We shall prove that for "large" switching costs firms end up charging the monopoly price ${ }^{4}$. When at least one of the switching costs is "small" we prove that there is no pure strategy equilibrium and characterize two different types of mixed strategy equilibria. First, we characterize single sided poaching equilibrium, where there is a positive probability that one firm charges a low price and captures the whole market, with the other firm unable to retaliate. Next we study double sided poaching equilibria, where both firms capture the whole market with positive probability.

\subsubsection{Large Switching Costs $\left(B_{i}, B_{j} \geq \frac{1}{2}\right)$}

In this case there is a pure strategy equilibrium that is easy to characterize and is intuitively appealing.

Theorem 3.2. If $B_{i}, B_{j} \geq \frac{1}{2}$ then in equilibrium both firm charge the monopoly price $\left(p_{1}=p_{2}=1\right)$.

\section{Idea of Proof:}

The intuition for this equilibrium is simple. Since the switching costs are so high, for a firm to poach on its rival's consumers, it must reduce prices drastically. The payoff received with this price reduction leaves firms worse off than charging the maximum price to their own inherited market share. Therefore in equilibrium firms charge the monopoly price. See 6.1 in the Appendix for details.

This assumption $\left(B_{i}, B_{j} \geq \frac{1}{2}\right)$ is equivalent to the typical assumption made in the switching costs literature: the switching cost is so high that firms do not have incentives to poach their rival's customers.

\footnotetext{
${ }^{4}$ This type of equilibrium is the commonly used in models where consumers are confronted with switching costs; the idea is that the switching cost is so high that it is unfeasible for consumers to change firms and therefore the firms are able to charge the monopoly price.
} 


\subsubsection{At Least One Small Switching Cost $\left(B_{i} \leq \frac{1}{2}\right.$ or $\left.B_{j} \leq \frac{1}{2}\right)$}

We first restate the Bertrand equilibrium in the context of our model.

Proposition 3.3. If $B_{i}, B_{j}=0$ then $p_{i}=p_{j}=0$

Now we show that the nature of the equilibria when at least one switching cost is low is completely different to when both switching costs are large. In fact, here equilibria will always involve randomization.

Proposition 3.4. If $\min \left\{B_{i}, B_{j}\right\}<\frac{1}{2}$ and $\max \left\{B_{i}, B_{j}\right\}>0$ then there does not exist a Nash equilibrium in pure strategies.

\section{Idea of Proof:}

The idea behind the proof is that for any pair of prices $\left(p_{i}^{*}, p_{j}^{*}\right)$ at least one of the firms has incentives to modify its price. Since one of the firms has a "small" switching cost, if it decides to charge a high price its rival will have incentives to poach its customers. Conversely, if the firm with a "small" switching cost imposes a low price so as to not risk losing his market share, then its rival will put a high price, since it is unable to poach, giving the first firm leeway to raise its price. This trade off is the essential reason as to why there is no pure strategy equilibrium. See 6.2 in the Appendix for details.

In order to characterize the mixed strategy equilibria we extend the methodology introduced in [15] by Shilony to the case of asymmetric switching costs that must be paid to firms (as opposed to symmetric switching costs that are deadweight lost).

Denote by $F_{i}, F_{j}$ the price distribution (i.e., the strategy) chosen by each firm. Given our assumption that consumers always purchase the good when the price is less than one and that consumers inherited by firm $i$ will only switch to $j$ if $p_{i}>p_{j}+B_{i}$ we have the following expression the firm $j$ 's expected payoff,

$$
\begin{aligned}
\mathbb{E}\left(\pi_{j}\left(p, p_{i}\right)\right) & =\int_{0}^{1} \pi_{j}(p, x) d F_{i}(x) \\
& =\int_{0}^{p-B_{j}} \frac{1}{2} B_{j} d F_{i}(x)+\int_{p-B_{j}}^{p+B_{i}} \frac{1}{2} p d F_{i}(x)+\int_{p+B_{i}}^{1} p d F_{i}(x) \\
& =F_{i}\left(p-B_{j}\right) \frac{1}{2}\left(B_{j}-p\right)-F_{i}\left(p+B_{i}\right) \frac{1}{2} p+p
\end{aligned}
$$

The first term in the second line corresponds to the case where firm $i$ 's price is so low that all of $j$ 's consumers decide to switch (and firm $j$ receives the consumers switching cost). The second term expresses the payoff $j$ receives in case that both firms retain their market share. And finally the third term reflects $j$ 's payoff when it manages to capture the entire market. For a given strategy of firm $i\left(F_{i}\right)$, we have that for every price in the support of $d F_{j}$ (firm $j$ 's strategy), $j$ 's payoff is a constant equal to $V_{j}$. Therefore we have the following equality, 


$$
\mathbb{E}\left(\pi_{j}\left(p, p_{i}\right)\right)=F_{i}\left(p-B_{j}\right) \frac{1}{2}\left(B_{j}-p\right)-F_{i}\left(p+B_{i}\right) \frac{1}{2} p+p=V_{j} \quad \forall p \in \operatorname{sop}\left(d F_{j}\right) .
$$

To derive the firms' strategies we shall consider a pair of auxiliary variables that represent the maximum and the minimum price that firm $i$ uses with a positive probability, $\bar{p}_{i}$ and $p_{i}$ respectively. ${ }^{5}$ Before we derive the firms' strategies in these cases we shall prove two technical Lemmas that characterize the minimum price a firm will use and the features of a firms strategy near that price.

Lemma 3.5. If $\min \left\{B_{i}, B_{j}\right\}<\frac{1}{2}$ and $\max \left\{B_{i}, B_{j}\right\}>0$ then $\underline{p}_{i}$ is positive.

\section{Proof:}

See 6.3 in Appendix for details.

Lemma 3.6. If $\min \left\{B_{i}, B_{j}\right\}<\frac{1}{2}$ then $F_{i}$ is continuous in $\underline{p}_{i}(i$ 's minimum price).

\section{Proof:}

For the case $B_{j}>0$ see 6.4 in Appendix for details. For the case in which $B_{j}=0$ the proofs may be found in 6.10 and 6.11.

Definition 3.7. We will say that firm $j$ poaches on firm $i$ 's customer base if the equilibrium strategies are such that

$$
\bar{p}_{i}-\underline{p}_{j}>B_{i}
$$

In such a case, with positive probability firm $j$ will gain firm $i$ 's consumers.

Single Sided Poaching Equilibrium. Let us first characterize the equilibrium in which only one firm may poach consumers from its rival. We call this type of equilibrium a single sided poaching equilibrium. In this case we characterize both firms' strategies and are able to find explicit expressions for $V_{i}$ and $V_{j}$.

Theorem 3.8. If the equilibrium strategies are such that there is single sided poaching, that is

I) $\bar{p}_{i}-\underline{p}_{j}>B_{i}$

II) $\bar{p}_{j}-\underline{p}_{i}<B_{j}$

then firm $j$ 's pricing strategy has the following cumulative distribution,

$$
F_{j}(p)= \begin{cases}0 & p<\underline{p}_{j} \\ \left(1-\frac{2 V_{i}-B_{i}}{p}\right) & \underline{p}_{j} \leq p<\bar{p}_{i}-B_{i} \\ \left(1-\frac{2 V_{i}-B_{i}}{\bar{p}_{i}-B_{i}}\right) & \bar{p}_{i}-B_{i} \leq p<\bar{p}_{j} \\ 1 & p \geq \bar{p}_{j}\end{cases}
$$

\footnotetext{
${ }^{5}$ These variables are also defined in Shilony [15], but in that model assumes that both firms have the same maximum and minimum price since the switching costs are identical. However we characterize asymmetric equilibria where the switching costs are the same, but the minimum prices are not.
} 
and firm i's pricing strategy has the following cumulative distribution

$$
F_{i}(p)= \begin{cases}0 & p<\underline{p}_{i} \\ 2\left(1-\frac{V_{j}}{p-B_{i}}\right) & \underline{p}_{j}+B_{i} \leq p<\bar{p}_{i} \\ 1 & p \geq \bar{p}_{i}\end{cases}
$$

with $\underline{p}_{i}=B_{i}+\frac{1}{2}, \underline{p}_{j}=\frac{1}{2}, \bar{p}_{i}=\bar{p}_{j}=1$ and the firms' expected payoff are,

$$
V_{i}=\frac{B_{i}}{2}+\frac{1}{4}, \quad V_{j}=\frac{1}{2}
$$

\section{Idea of Proof:}

Notice that the price difference between $j$ 's maximum price and $i$ 's minimum price is smaller than $B_{j}$. Therefore $i$ never imposes a price that attracts $j$ 's consumers. This means that the firms' equations for their expected payoffs take on a simpler form since,

$$
p+B_{j}>\bar{p}_{j} \quad \forall p \in \operatorname{sop}\left(d F_{i}\right) \quad \text { and } \quad p-B_{j}<\underline{p}_{i} \quad \forall p \in \operatorname{sop}\left(d F_{j}\right)
$$

therefore equation 3.2 takes on the following form,

$$
\begin{gathered}
F_{j}\left(p-B_{i}\right) \frac{1}{2}\left(B_{i}-p\right)+\frac{1}{2} p=V_{i} \quad \forall p \in \operatorname{sop}\left(d F_{i}\right) \\
-F_{i}\left(p+B_{i}\right) \frac{1}{2} p+p=V_{j} \quad \forall p \in \operatorname{sop}\left(d F_{j}\right) .
\end{gathered}
$$

Given the nature of the firms' expected payoff equations and using the fact that $F_{i}$ and $F_{j}$ are continuous in $\underline{p}_{i}$ and $\underline{p}_{j}$ respectively, we can deduce that $\underline{p}_{i}=\underline{p}_{j}+B_{i}$. Then, using the firms' expected payoff equations, we find the functional form of the firms' strategies. Finally, we impose the condition that the firms' payoff must be lower for prices which are not in the support of their strategies, and are able to find expressions for $\bar{p}_{i}, \underline{p}_{i}, \bar{p}_{j}, \underline{p}_{j}, V_{i}$, and $V_{j}$, giving us the equilibrium described in the Theorem. See 6.5 in the Appendix for details of proof.

An example of the firms pricing strategy in this equilibrium can be see in Figure 4.

Corollary 3.9. Such an equilibrium exists if and only if $B_{i}<\frac{1}{2}$ and $B_{i}+B_{j}>\frac{1}{2}$.

\section{Proof:}

Replace values for $\underline{p}_{i}, \underline{p}_{j}, \bar{p}_{i}$ and $\bar{p}_{j}$ in conditions $I$ and $I I$ of Theorem 3.8.

Observation: this result proves that even when we consider symmetrical switching costs $B_{i}=B_{j}$, we can have asymmetrical equilibria, where only one of the firms poaches its' rivals customers. This type of equilibria is not considered in Shilony's symmetrical switching cost model [15]. 
The regions where these equilibrium exist can be seen in Figure 5 and the firms expected payoff can be seen graphically in Figure 6.

This completes the study of equilibria in which only one firm has the possibility to attract its rival's consumers. Now we must find the equilibria in which both firms may poach customers.

Double Sided Poaching Equilibrium If both firms are to poach with possible probability we must have

$$
\begin{gathered}
\bar{p}_{i}-\underline{p}_{j}>B_{i} \\
\bar{p}_{j}-\underline{p}_{i}>B_{j} .
\end{gathered}
$$

In other words $j$ has the possibility to attract $i$ 's consumers and vice versa. We will focus on the characterization of equilibria such that,

$$
\bar{p}_{i}-\underline{p}_{i} \leq B_{i}+B_{j}
$$

This condition tells us that the range of possible prices that a firm imposes is not too large compared to the switching costs. We obtain different types of equilibrium strategies when this last inequality holds with equality or with strict inequality.

The next two Lemmas characterize the relevant features of the equilibria for different cases of this last inequality.

Lemma 3.10. If the equilibrium strategies are such that there is double sided poaching and that

$$
\begin{aligned}
& \text { I) } \bar{p}_{i}-\underline{p}_{i}<B_{i}+B_{j} \\
& \text { II) } \bar{p}_{j}-\underline{p}_{j} \leq B_{i}+B_{j}
\end{aligned}
$$

then firm $j$ 's price has the following cumulative distribution,

$$
F_{j}(p)= \begin{cases}0 & p<\underline{p}_{j} \\ \left(1-\frac{2 V_{i}-B_{i}}{p}\right) & \underline{p}_{j} \leq p<\bar{p}_{i}-B_{i} \\ \left(1-\frac{2 V_{i}-B_{i}}{\bar{p}_{i}-B_{i}}\right) & \bar{p}_{i}-B_{i} \leq p<\underline{p}_{i}+B_{j} \\ 2\left(1-\frac{V_{i}}{p-B_{j}}\right) & \underline{p}_{i}+B_{j} \leq p<\bar{p}_{j} \\ 1 & p \geq \bar{p}_{j}\end{cases}
$$

with $\underline{p}_{i}=2 V_{j}-B_{j}, \bar{p}_{i}=1$ and the firms' expected payoff satisfy the following equation,

$$
4 V_{i} V_{j}-2 V_{i} B_{j}+2 V_{i} B_{i}+2 V_{j}-2 V_{i}-B_{j}-4 V_{j} B_{i}+2 B_{i} B_{j}=0
$$




\section{Idea of Proof:}

Given $(I)$ and $(I I)$ we can see the partition of $\operatorname{sop}\left(d F_{i}\right)$ and $\operatorname{sop}\left(d F_{j}\right)$ in Figure 1. The methodology consists of using equation 3.2 and considering $p$ in the different intervals defined in the hypothesis of the Lemma, so we can derive a functional form for $F_{j}$ that makes 3.2 hold. By considering $p$ in different intervals, equation 3.2 takes on a simpler form, canceling either $F_{j}\left(p-B_{i}\right)$ or $F_{j}\left(p+B_{j}\right)$, which allows us to find an expression for $F_{j}\left(p+B_{j}\right)$ and $F_{j}\left(p-B_{i}\right)$ respectively.

With this process, we can find the complete functional form for $F_{j}$, which depends on $\underline{p}_{i}, \bar{p}_{i}, V_{i}$, and $V_{j}$. In this Lemma we find relationships between these variables. To do this, we impose necessary conditions on equation 3.2 and $F_{j}$ so that they correspond to $i$ 's expected payoff equation and $j$ 's pricing strategy distribution, respectively. In this case the conditions that must be imposed are that $F_{i}$ is continuous in $\underline{p}_{i}$, that if $p$ is higher than $\bar{p}_{i}$ or lower than $\underline{p}_{i}$ there must be a reduction in firm $i$ 's expected payoff, and that $F_{j}$ must be increasing. By imposing these conditions we get the values and relationships for the variables described in the Lemma. See 6.6 in Appendix for details.

An example of the firms' pricing strategy in this equilibrium can be see in Figure 4.

Note that Lemma 3.10 not only tells us the relationship that $V_{i}$ and $V_{j}$ must satisfy in this type of equilibrium, but it also gives us an expression for the distribution of prices used by $j$. Now we must analyze the case in which a firm's price spread is equal to the sum of both switching costs.

Lemma 3.11. If the equilibrium strategies are such that there is double sided poaching and that

$$
\begin{aligned}
& \text { I) } \bar{p}_{i}-\underline{p}_{i}=B_{i}+B_{j} \\
& \text { II) } \bar{p}_{j}-\underline{p}_{j} \leq B_{i}+B_{j}
\end{aligned}
$$

then firm $j$ 's price has the following cumulative distribution,

$$
F_{j}(p)= \begin{cases}0 & p<\underline{p}_{j} \\ \left(1-\frac{2 V_{i}-B_{i}}{p}\right) & \underline{p}_{j} \leq p<\bar{p}_{i}-B_{i} \\ 2\left(1-\frac{V_{i}}{p-B_{j}}\right) & \underline{p}_{i}+B_{j} \leq p<\bar{p}_{j} \\ 1 & p \geq \bar{p}_{j}\end{cases}
$$

with $\underline{p}_{i}=2 V_{j}-B_{j}, \bar{p}_{i}=2 V_{j}+B_{i}$ and the firms' expected payoff satisfy the following equation,

$$
4 V_{j}^{2}-2 V_{j} B_{j}-2 V_{i} B_{j}-2 V_{j} B_{i}+B_{i} B_{j}=0
$$

\section{Idea of Proof:}

As with Lemma 3.10, we must use the hypothesis to divide $\operatorname{sop}\left(d F_{i}\right)$ and $\operatorname{sop}\left(d F_{j}\right)$ into smaller intervals where we can derive $F_{j}$ 's functional form using equation 3.2 (see Figure 2). As before, this is done by canceling out the expressions for $F_{j}\left(p-B_{i}\right)$ or $F_{j}\left(p+B_{j}\right)$ (depending on the interval of $\operatorname{sop}\left(d F_{i}\right)$ that we consider) to solve $F_{j}$. Once we have determined $F_{j}$ 's functional form we use Lemma 3.6 to find a relationship between $\underline{p}_{i}$ and $V_{j}$ and we impose the necessary conditions that 3.2 must satisfy. Specifically, 
that if we consider $p$ larger or smaller than $\bar{p}_{i}$ and $\underline{p}_{i}$ respectively, there must be a reduction in the firm's expected payoff. Using these arguments we get the expressions for $\bar{p}_{i}, \underline{p}_{i}$, and the equation that $V_{i}$ and $V_{j}$ must satisfy. See 6.7 in Appendix for details.

An example of the firms' pricing strategy in this equilibrium can be see in Figure 4.

Now that we have found the firms' strategies and the conditions that their expected payoffs must satisfy, we can examine the types of double sided poaching equilibria that arise. We must solve the system of equations that the firms' expected payoff satisfy to find the particular expression that those payoffs take. We have three types of double sided poaching equilibria to consider:

Theorem 3.12. The optimal strategies characterized in Lemmas 3.10 and 3.11 give the following equilibria,

Case I: $\bar{p}_{i}-\underline{p}_{i}<B_{i}+B_{j}$ and $\bar{p}_{j}-\underline{p}_{j}<B_{i}+B_{j}$.

$$
V_{i}=\frac{1}{4}\left(\frac{3 B_{i}+B_{j}-\left(B_{i}+B_{j}\right)^{2}+\xi\left(B_{i}, B_{j}\right)}{2-B_{i}-B_{j}}\right)
$$

and

$$
V_{j}=\frac{1}{4}\left(\frac{3 B_{j}+B_{i}-\left(B_{i}+B_{j}\right)^{2}+\xi\left(B_{i}, B_{j}\right)}{2-B_{i}-B_{j}}\right)
$$

where $\xi(x, y)$ takes the following expression, ${ }^{6}$

$$
\begin{aligned}
\xi(x, y)= & \left(-26 x y-11 x^{2}-11 y^{2}+22 x y^{2}+22 x^{2} y+2 x^{3}+2 y^{3}-\right. \\
& \left.4 x y^{3}-10 x^{2} y^{2}-4 x^{3} y+x^{4}+y^{4}+8 x+8 y\right)^{\frac{1}{2}}
\end{aligned}
$$

Case II: $\bar{p}_{i}-\underline{p}_{i}<B_{i}+B_{j}$ and $\bar{p}_{j}-\underline{p}_{j}=B_{i}+B_{j}$.

$$
V_{i}=\frac{1}{4}\left(3 B_{i}+B_{j}-1+\alpha\left(B_{i}, B_{j}\right)\right)
$$

and

$$
V_{j}=\frac{1}{4}\left(1+B_{j}-B_{i}+\left(2 B_{i}-1\right) \alpha\left(B_{i}, B_{j}\right)\right)^{\frac{1}{2}}
$$

where $\alpha(x, y)$ takes the following expression,

$$
\alpha(x, y)=\left(y^{2}-2 x y-3 x^{2}+2 x+2 y+1\right)^{\frac{1}{2}}
$$

Case III: $\bar{p}_{i}-\underline{p}_{i}=B_{i}+B_{j}$ and $\bar{p}_{j}-\underline{p}_{j}=B_{i}+B_{j}$.

$$
V_{i}=\frac{(1+\sqrt{5}) B_{i}+2 B_{j}}{4}
$$

and

$$
V_{j}=\frac{(1+\sqrt{5}) B_{j}+2 B_{i}}{4} .
$$

\footnotetext{
${ }^{6}$ Note that $\xi(x, y)=\xi(y, x)$.
} 


\section{Idea of Proof:}

We just combine the results of Lemmas 3.10 and 3.11. See 6.8 in Appendix for details.

Corollary 3.13. The partition of the switching cost space $\left(B_{i}, B_{j}\right)$ when we consider double sided poaching equilibria is defined by the following bounds:

Case I: $1-\left(2 V_{j}-B_{j}\right)<B_{i}+B_{j}$ and $1-\left(2 V_{i}-B_{i}\right)<B_{i}+B_{j}$ where $V_{i}$ and $V_{j}$ take on the values expressed in Case I of Theorem 3.12.

Case II: $1-\left(2 V_{j}-B_{j}\right)<B_{i}+B_{j}$ and $2 V_{i}+B_{j} \leq 1$ where $V_{i}$ and $V_{j}$ take on the values expressed in Case II of Theorem 3.12.

Case III: $2 V_{j}+B_{i}<1$ and $2 V_{i}+B_{j} \leq 1$ where $V_{i}$ and $V_{j}$ take on the values expressed in Case III of Theorem 3.12 .

\section{Proof:}

The bounds in case $I$ are derived by replacing the expressions for $\bar{p}_{i}, \underline{p}_{i}, \bar{p}_{j}$, and $\underline{p}_{j}$ found in Lemma 3.10 in the inequalities $\bar{p}_{i}-\underline{p}_{i}<B_{i}+B_{j}$ and $\bar{p}_{j}-\underline{p}_{j}<B_{i}+B_{j}$. Then we replace the values of $V_{i}$ and $V_{j}$ for case $I$ found in Theorem 3.12 to characterize the corresponding subset of $\left(B_{i}, B_{j}\right)$. The bounds in case $I I$ are derived by replacing the expressions for $\bar{p}_{i}$ and $\underline{p}_{i}$ found in Lemma 3.10 into inequality $\bar{p}_{i}-\underline{p}_{i}<B_{i}+B_{j}$ and by replacing the expression for $\bar{p}_{j}$ found in Lemma 3.11 to the inequality $\bar{p}_{j} \leq 1^{7}$. Then we replace the values of $V_{i}$ and $V_{j}$ for case $I I$ found in Theorem 3.12 to characterize the corresponding subset of $\left(B_{i}, B_{j}\right)$. Finally, for case $I I I$ we replace the values of $\bar{p}_{i}$ and $\bar{p}_{j}$ found in Lemma 3.11 in the inequalities $\bar{p}_{i} \leq 1$ and $\bar{p}_{j} \leq 1$. Then we replace the values of $V_{i}$ and $V_{j}$ found in Theorem 3.12 to characterize the corresponding subset of $\left(B_{i}, B_{j}\right)$.

The partition defined by Corollary 4 can be seen in Figure 5 and the firms' corresponding expected payoffs in Figures 7, 8, and 9.

With this final result we know exactly what the equilibria are when partially "locked-in" consumers are faced with any pair of switching costs. In the next section we shall discuss the nature of these equilibria and explain the economic intuition behind them. To close this section we show that there is no equilibrium with $\bar{p}_{i}-\underline{p}_{i}>B_{i}+B_{j}$ such the firms strategies have a connected support. ${ }^{8}$

Theorem 3.14. There does not exist a Nash equilibrium in which

$$
\bar{p}_{i}-\underline{p}_{i}>B_{i}+B_{j}
$$

with a connected support.

\footnotetext{
${ }^{7}$ Here we impose the condition that that the maximum price must be less than 1.

${ }^{8}$ Note that Shilony's proof of uniqueness for the equilibria derived in [15] does not consider the possibility of strategies without connected support.
} 


\section{Idea of Proof:}

To prove this result we must consider two separate cases,
a) $\bar{p}_{j}-\underline{p}_{j}<2\left(B_{i}+B_{j}\right)$
b) $\bar{p}_{j}-\underline{p}_{j} \geq 2\left(B_{i}+B_{j}\right)$

and as with the other Lemmas in this section we must consider the properties that the firms' strategies must satisfy. Specifically for case $a$ ), we consider an interval of feasible strategies and find that we get two incompatible expressions for a firm's mixed strategy. This leads to a contradiction.

A similar analysis applies to case $b$ ) where we consider a large price spread for $j$ 's strategy. This large price spread allows us to deduce that $i$ 's price spread must be smaller than $2\left(B_{i}+B_{j}\right)$, in which case we fall under case $a$ )'s assumptions, which we already proved to be false, giving us a contradiction. See 6.9 in Appendix for details.

\section{Discussion of Equilibria}

In the previous section we characterized the equilibria that can arise for all possible levels of $\left(B_{i}, B_{j}\right)$. For the case in which one firms inherits the entire market we have that the dominant firm charges the switching cost that his consumers face, while the rivaling firm is left out of the market. When both firms inherit half of the market the results are very sensitive to the size of the switching costs. If both firms have relatively high switching costs $\left(B_{i}, B_{j} \geq \frac{1}{2}\right.$, characterized in Theorem 3.2) there is a pure strategy equilibrium where both firms charge the monopoly price and continue to split the market. This occurs because the firms do not fear the loss of their client base, thus giving them the liberty to charge the consumers' reserve price to maximize their profits. This result is in line with the typical assumption made in many switching cost models, in which this cost is so high that consumers never switch, eliminating any form of competition.

When one of the firms' switching cost is relatively low there is no pure strategy equilibrium, since the the firms can either charge high prices to "milk" their own consumers or charge a low price to "poach" their rivals customer base. This tradeoff forces us to find mixed strategy equilibria which can basically take two forms. What we call single sided poaching corresponds to equilibria where only one firm is able to capture the entire market with positive probability. In this case only that firm puts positive probability on low prices while the other concentrates in milking its inherited customer base. The non poaching firm may post prices below the monopoly price, but the purpose is to protect itself from losing market share, and does not intend to expand it. An interesting outcome of this equilibrium is that the payoff of the poaching firm is independent of $B_{i}$ and $B_{j}$, and equal to the payoff received when it charges the 
monopoly price. The payoff received by the firm that cannot poach consumers depends on the value of his own switching cost and is lower than the monopoly payoff. The firms' expected payoff for the resulting equilibria can be seen in Figure 6.

We denote by double sided poaching the case in which both firms are able to capture the entire market with positive probability. For these equilibria the firms' resulting strategies consist of two intervals (which may or may not be connected). One of these intervals (high prices) corresponds to price levels that firms use to "milk" their customer base and the other (low prices) corresponds to price levels where firms try to capture the entire market. In the characterization of the double sided poaching equilibria we must consider three different types of equilibria, which depend strongly on the range of the support of their strategies. The firms' expected payoff for these three types of equilibria can be seen in Figures 7, 8, and 9 .

With this, we have characterized equilibria for any values of $\left(B_{i}, B_{j}\right) \in[0,1]^{2}$, these different resulting equilibria can be seen in Figure 5. It is important to mention that for some of the values of $\left(B_{i}, B_{j}\right)$ there is more than one equilibrium. We do not find any reason why one equilibrium should be selected over another and the correct choice of a refinement will depend on the concrete application being considered.

\section{Conclusions}

In this paper we study the effects that asymmetric switching costs can have on firms' strategies and payoffs. We introduce a simple model, where two identical firms have inherited a fraction of the market. Consumers who are inherited by firm $i$ face a cost of switching to firm $j$ that is, in general, different from the cost of switching of a consumer inherited by firm $j$. In our model, these switching costs are paid out to the firm that the consumer leaves, and are not deadweight lost (as in, for example, Shilony [15]).

For any combination of switching costs we characterize at least one resulting equilibrium. In many cases multiple equilibria may exist. In the case of high switching costs, firms act as a monopoly over their customer base, eliminating any benefits from price competition. This is the case assumed in most of the switching cost literature, which considers exogenously high switching costs that eliminate any possibility for consumers to change their decisions. When we move to analyze the case of smaller switching costs, the firms market power dissipates and at least one of the firms' payoff is reduced. Pure strategy equilibria do not exist, since firms confront a stark tradeoff between charging a high price to their own customers (which are, to a certain degree, locked in), or pricing aggressively to try to capture their rival's market share. These results extend the analysis documented in Shilony [15] to the case of asymmetric switching costs that must be paid out to the firm from which the consumer switches.

The main contribution of this paper is the fact that we consider asymmetric switching costs be- 
tween firms, and find that small differences in these switching costs may completely change the nature of the equilibrium. We consider this to be an essential stage of a full model where switching costs are strategically chosen in a previous stage of the game. The ultimate goal of such a model is to understand the implications that endogenously chosen switching costs may have on the degree of competition between two identical firms. The equilibriums characterized in this paper will be the resulting equilibrium for the final period of such a multi-period model, allowing for a complete characterization of subgame perfect equilibria. The open question is then posed: if we consider completely rational consumers that are forward looking and internalize all of the available information, does the possibility of giving gifts (which later on become switching costs) affect competition? This issue will be addressed in future work that depends on the results found on this paper. 


\section{Appendix}

\subsection{Proof of Theorem 3.2 :}

We will show that these prices

$$
p_{2 i}^{*}=p_{2 j}^{*}=1
$$

are an equilibrium. With these price levels we have $\pi_{2 i}=\pi_{2 j}=\frac{1}{2}$.

If firm $i$ chooses a lower price to capture the entire market it receives the following payoff,

$$
\pi_{2 i}^{\prime}=1-B_{j}-\epsilon \leq \frac{1}{2}
$$

since $B_{j} \geq \frac{1}{2}$. Therefore it does not have incentives to lower its price.

\subsection{Proof of Proposition 3.4 :}

Without loss of generality, we assume that $B_{i}=\min \left\{B_{i}, B_{j}\right\}<\frac{1}{2}, B_{j}=\max \left\{B_{i}, B_{j}\right\}>0$, and $\left(p_{2 i}^{*}, p_{2 j}^{*}\right)$ a Nash equilibrium in pure strategies. We analyze 6 different cases and find a profitable deviation in each one,

(i) $0<p_{2 i}^{*}<p_{2 j}^{*}-B_{j}$ (in case that $p_{2 j}^{*}-B_{j}>0$ ): we have that $\pi_{2 i}=p_{2 i}^{*}$ and in that case firm $i$ has incentives to raise his price in $\epsilon$ so that he continues to have the entire market and raises his payoff.

(ii) $\max \left\{0, p_{2 j}^{*}-B_{j}\right\} \leq p_{2 i}^{*}<\min \left\{1, p_{2 j}^{*}+B_{i}\right\}$ : we have that $\pi_{i}=\frac{p_{2 i}^{*}}{2}$, in this case firm $i$ has incentives to raise his price in $\epsilon$ so that the he still retains his market share and raises his payoff.

(iii) $0=p_{2 i}^{*}=p_{2 j}^{*}+B_{i}$ : we have that both firms receive a payoff of zero. If $j$ raises his price in $\epsilon$ so that he maintains his market share, he will receive a positive payoff.

(iv) $0<p_{2 i}^{*}=p_{2 j}^{*}+B_{i} \leq 1$ : we have that the payoff for firm $j$ is $\pi_{2 j}=\frac{p_{2 j}^{*}}{2}$ and in this case he has incentives to reduce his price in $\epsilon$ to capture all of $i$ 's customers and have a payoff of $\pi_{2 j}=p_{2 j}^{*}-\epsilon$. For $\epsilon$ sufficiently small, this means a higher payoff for $j$.

(v) $p_{2 j}^{*}+B_{i}<p_{2 i}^{*} \leq 1$ : we have that $\pi_{2 j}=p_{2 j}^{*}$, in this case firm $j$ has incentives to raise his price in $\epsilon$ so that he still sells to the entire market but at a higher price.

(vi) $p_{2 i}^{*}=p_{2 j}^{*}=1$ : here we have that $j$ 's payoff is $\pi_{2}=\frac{1}{2}$ and in that case he has incentives to lower his price to $p_{2 j}^{*}=1-B_{i}-\epsilon$, and that way poach all of $i$ consumers and receive a payoff of $\pi_{2 j}=1-B_{i}-\epsilon>$ $\frac{1}{2}$ (for $\epsilon$ sufficiently small since $B_{i}<\frac{1}{2}$ ). 


\subsection{Proof of Lemma 3.5 :}

\section{Proof:}

Since $\min \left\{B_{i}, B_{j}\right\}<\frac{1}{2}$ we have a mixed strategy equilibrium with a maximum and minimum price for firm $i \mathrm{i}\left(\bar{p}_{i}, \underline{p}_{i}\right)$. We shall separate the proof in two cases: $B_{i} \neq 0$ and $B_{i}=0$. Let us first analyze $B_{i} \neq 0$. Looking at the firms utility function we know that in the worst case scenario, firm $i$ will have a payoff of $\frac{B_{i}}{2}>0{ }^{9}$. If $\underline{p}_{i}=0$, it's expected payoff in the second period is,

$$
\begin{aligned}
\mathbb{E}\left(\pi_{i}\left(0, p_{j}\right)\right) & =\int_{0-B_{i}}^{0+B_{j}} \frac{1}{2} 0 d F_{j}(x)+\int_{0+B_{j}}^{1} 0 d F_{j}(x) \\
& =0
\end{aligned}
$$

in other words, $V_{i}=0 \forall p \in \operatorname{sop}\left(d F_{i}\right)$. Which is a contradiction since we just noted that $V_{i}>0$.

Now we analyze the case when $B_{i}=0$. Given the hypothesis, we know that $B_{j}>0$. Again we assume that $\underline{p}_{i}=0$ and prove that this leads to a contradiction. If $\underline{p}_{i}=0$ then $V_{i}=0$ since every strategy price in the support has the same expected payoff. Suppose that firm $i$ fixes a price $\epsilon>0$, then his expected payoff is,

$$
\begin{aligned}
\mathbb{E}\left(\pi_{i}\left(\epsilon, p_{j}\right)\right) & =\int_{0}^{\epsilon} \frac{1}{2} 0+\int_{\epsilon}^{\epsilon+B_{j}} \frac{1}{2} \epsilon d F_{j}(x)+\int_{\epsilon+B_{j}}^{1} \epsilon d F_{j}(x) \\
& =-\frac{1}{2}\left(F_{j}\left(\epsilon+B_{j}\right)+F_{j}(\epsilon)\right)+\epsilon
\end{aligned}
$$

This last expression has two possible values. We have that in one case,

$$
F_{j}\left(\epsilon+B_{j}\right)+F_{j}(\epsilon)<2
$$

in which case we have that $-\frac{1}{2}\left(F_{j}\left(\epsilon+B_{j}\right)+F_{j}(\epsilon)\right)+\epsilon>0$, which is a contradiction since if $\underline{p}_{i}=0$, then $V_{i}=0$. In the other case we have,

$$
F_{j}\left(\epsilon+B_{j}\right)+F_{j}(\epsilon)=2
$$

which implies that $F_{j}(\epsilon)=1 \forall \epsilon>0$. Since $F_{j}$ is a cumulative distribution, we know that $F_{j}$ is continuous from the right. Taking limit for when $\epsilon \longrightarrow 0^{+}$we have that $F_{j}(0)=1$, this means that there is only one feasible price, $\operatorname{sop}\left(d F_{i}\right)=\{0\}$, and therefore we are in presence of a pure strategy equilibrium. This is a contradiction with Proposition 3.2.

\footnotetext{
${ }^{9}$ This occurs when firm $i$ loses his market share, and consumers must return the bond to purchase from $j$.
} 


\subsection{Proof of Lemma 3.6 part I:}

\section{Proof:}

In this part we analyze the case when $B_{j}>0$. Let us suppose that $F_{i}$ is not continuous in $\underline{p}_{i}$, in other words $F_{i}\left(\underline{p}_{i}\right)=a>0$ (obviously $F_{i}(p)=0 \forall p<\underline{p}_{i}$ ) and assume that firm $j$ imposes a price $p_{j}=\underline{p}_{i}{ }^{10}$. Therefore firm $i$ 's expected payoff takes the following form,

$$
\begin{aligned}
\mathbb{E}\left(\pi_{j}\left(\underline{p}_{i}, p_{i}\right)\right) & =\int_{0}^{1} \pi_{j}\left(\underline{p}_{i}, x\right) d F_{i}(x) \\
& =\int_{\underline{p}_{i}}^{\underline{p}_{i}+B_{i}} \frac{1}{2} \underline{p}_{i} d F_{i}(x)+\int_{\underline{p}_{i}+B_{i}}^{1} \underline{p}_{i} d F_{i}(x) \\
& =\frac{1}{2} \underline{p}_{i} F_{i}\left(\underline{p}_{i}+B_{i}\right)-\frac{1}{2} \underline{p}_{i} \underbrace{F_{i}\left(\underline{p}_{i}\right)}_{=a}+\underline{p}_{i} \underbrace{F_{i}(1)}_{=1}-\underline{p}_{i} F_{i}\left(\underline{p}_{i}+B_{i}\right) \\
& =\underline{p}_{i}-\frac{1}{2} \underline{p}_{i} F_{i}\left(\underline{p}_{i}+B_{i}\right)-\frac{1}{2} a \underline{p}_{i}
\end{aligned}
$$

This last expression must be equal to the right hand side of 3.1 when we make $p$ converge to $p$ from the right. Since $F_{i}$ is a cumulative distribution function and therefore continuous from the right, ${ }^{11}$

$$
\lim _{p \rightarrow \underline{p}_{i}^{+}} F_{i}\left(p-B_{j}\right) \frac{1}{2}\left(B_{j}-p\right)-F_{i}\left(p+B_{i}\right) \frac{1}{2} p+p=\underline{p}_{i}-\frac{1}{2} \underline{p}_{i} F_{i}\left(\underline{p}_{i}+B_{i}\right)
$$

This implies that $\frac{1}{2} a \underline{p}_{i}=0$ and since we know from Lemma 3.5 that $\underline{p}_{i}>0$, we have a contradiction.

\subsection{Proof of Theorem 3.8:}

The methodology consists in finding the firms strategies via equation 3.2 and then imposing necessary conditions upon the maximum and minimum prices so that we are able to find the firms expected payoff. In this case, thanks to hypothesis $(I)$ and $(I I)$, equation 3.2 takes on a particular form for each firm. The firms expected payoff satisfy the following equations,

$$
\begin{aligned}
\underbrace{F_{i}\left(p-B_{j}\right)}_{=0, \text { since } p-B_{j}<\underline{p}_{i}}\left(\frac{1}{2}\left(B_{j}-p\right)\right)-F_{i}\left(p+B_{i}\right) \frac{1}{2} p+p & =V_{j} \\
-F_{i}\left(p+B_{i}\right) \frac{1}{2} p+p & =V_{j}
\end{aligned}
$$

\footnotetext{
${ }^{10}$ It is possible that $\underline{p}_{i} \notin \operatorname{sop}\left(d F_{j}\right)$, but this only implies that equation 3.2 does not hold, which is not relevant for the Lemmas proof.

${ }^{11}$ Note that since $B_{j}>0$ we have that $F_{i}\left(\underline{p}_{i}-B_{j}\right)=0$.
} 
and

$$
\begin{aligned}
F_{j}\left(p-B_{i}\right)\left(\frac{1}{2}\left(B_{i}-p\right)\right)-\underbrace{F_{j}\left(p+B_{j}\right)}_{=1, \text { since } p+B_{j}>\bar{p}_{j}} \frac{1}{2} p+p & =V_{i} \\
F_{j}\left(p-B_{i}\right)\left(\frac{1}{2}\left(B_{i}-p\right)\right)+\frac{1}{2} p & =V_{i}
\end{aligned}
$$

What simplifies the analysis is the fact that the term in equation 3.2 that contains $B_{j}$ disappears. Let us analyze the firms strategies separately. First, for firm $j$, if we consider $p^{*}>\bar{p}_{i}-B_{i}$ we have that $F_{i}\left(p^{*}+B_{i}\right)=1$, therefore equation 6.1 turns into $\frac{1}{2} p^{*}=V_{j}$, which means that $p^{*} \notin \operatorname{sop}\left(d F_{j}\right)$, since $V_{j}$ must be constant for every feasible $p^{*}$. Therefore, we have $F_{j}\left(p^{*}\right) \equiv K$ for all $p^{*}>\bar{p}_{i}-B_{i}$.

Using the same argument for $i$, if we consider $p^{\prime}<\underline{p}_{j}+B_{i}$, then we have that $F_{j}\left(p^{\prime}-B_{i}\right)=0$ which turns equation 6.2 into $\frac{1}{2} p^{\prime}=V_{i}$. Again, this means that $p^{\prime}$ cannot be in $\operatorname{sop}\left(d F_{i}\right)$ because if so $V_{i}$ would no be constant. Therefore we have that $F_{i}(p) \equiv K$ for all $p^{\prime}<\underline{p}_{j}+B_{i}$.

Using Lemma 3.6 we have that $F_{j}$ and $F_{i}$ are continuous in their respective minimum prices. Therefore we have that $\underline{p}_{i}=\underline{p}_{j}+B_{i}$ because since $F_{i}(p)$ is constant for $p<\underline{p}_{j}+B_{i}$, the only possible value for that constant is zero. Then $\underline{p}_{i}<\underline{p}_{j}+B_{i}$ and there would be no chance for $F_{i}$ to be continuous in $\underline{p}_{i}$. Therefore we have,

$$
\begin{gathered}
F_{i}(p)=0 \quad \forall p<\underline{p}_{j}+B_{i}, \quad \text { and } \\
\underline{p}_{i}=\underline{p}_{j}+B_{i}
\end{gathered}
$$

This last relationship is very intuitive. Since $i$ cannot poach from $j$, it does not have any incentives to put a low price. Therefore, the minimum price it uses in equilibrium is the one where $j$ could possibly begin to poach $i$ 's consumers.

Let us consider $p^{*}<\bar{p}_{i}-B_{i}$ so that $F_{i}\left(p+B_{i}\right)$ is not constant in equation 6.1 therefore,

$$
\begin{aligned}
-F_{i}\left(p^{*}+B_{i}\right) \frac{1}{2} p^{*}+p^{*} & =V_{j} \\
F_{i}\left(p^{*}+B_{i}\right) & =2\left(1-\frac{V_{j}}{p^{*}}\right) \quad \forall p^{*} \in\left[\underline{p}_{j}, \bar{p}_{i}-B_{j}\right]
\end{aligned}
$$

which gives us,

$$
F_{i}(p)=2\left(1-\frac{V_{j}}{p-B_{i}}\right) \quad \forall p \in\left[\underline{p}_{j}+B_{i}, \bar{p}_{i}\right]
$$


If we consider $p^{\prime}>\underline{p}_{j}+B_{i}$, using the same argument we can find an expression for $F_{j}$ from 6.2,

$$
F_{j}(p)=\left(1-\frac{2 V_{i}-B_{i}}{p}\right) \quad \forall p \in\left[\underline{p}_{j}, \bar{p}_{i}-B_{i}\right]
$$

Thus we have the following expressions for $F_{j}$ and $F_{i}$,

$$
F_{j}(p)= \begin{cases}0 & p<\underline{p}_{j} \\ \left(1-\frac{2 V_{i}-B_{i}}{p}\right) & \underline{p}_{j} \leq p<\bar{p}_{i}-B_{i} \\ \left(1-\frac{2 V_{i}-B_{i}}{\bar{p}_{i}-B_{i}}\right) & \bar{p}_{i}-B_{i} \leq p<\bar{p}_{j} \\ 1 & p \geq \bar{p}_{j}\end{cases}
$$

and

$$
F_{i}(p)= \begin{cases}0 & p<\underline{p}_{i} \\ 2\left(1-\frac{V_{j}}{p-B_{i}}\right) & \underline{p}_{j}+B_{i} \leq p<\bar{p}_{i} \\ 1 & p \geq \bar{p}_{i}\end{cases}
$$

Using Lemma 3.6 we can find relations between $\underline{p}_{i}, \underline{p}_{j}$ and $V_{j}, V_{i}$. In fact,

$$
\begin{aligned}
\lim _{p \rightarrow \underline{p}_{i}^{+}} F_{i}(p) & =2\left(1-\frac{V_{j}}{\underline{p}_{i}-B_{i}}\right)=0 \\
\Longrightarrow \underline{p}_{i} & =V_{j}+B_{i}
\end{aligned}
$$

and

$$
\begin{aligned}
\lim _{p \rightarrow \underline{p}_{j}^{+}} F_{j}(p) & =\left(1-\frac{2 V_{i}-B_{i}}{\underline{p}_{j}}\right)=0 \\
\Longrightarrow \underline{p}_{j} & =2 V_{i}-B_{i}
\end{aligned}
$$

With these relationships and equation 6.3, we can write,

$$
V_{j}+B_{i}=2 V_{i}
$$

Now let us see the conditions that must be imposed on the minimum and maximum prices. First, consider $p^{*} \geq \bar{p}_{i}$, we must have a reduction in expected payoff for firm $i$ in equation 6.2. Given the expression we have for $F_{j}$, we know that $F_{j}\left(p^{*}-B_{i}\right) \equiv K$, therefore we have

$$
\frac{1}{2} K\left(B_{i}-p^{*}\right)+\frac{1}{2} p^{*} \leq V_{i}
$$


Since the left hand side of the above equation is increasing in $p^{*}$, therefore get $\bar{p}_{i}=1$. In a similar manner, if we consider $p^{\prime}>\bar{p}_{j}$, we must have

$$
-\frac{1}{2} p^{\prime}+p^{\prime}=\frac{1}{2} p^{\prime} \leq V_{j}
$$

which means that $\bar{p}_{j}=1$ since the left hand side is increasing in $p^{\prime}$. Now, evaluating 6.1 in $\bar{p}_{j}=1$, we get an expression for $V_{j}$,

$$
\frac{1}{2} \bar{p}_{j}=\frac{1}{2}=V_{j}
$$

and using these values, combined with equation 6.5 we have that $V_{i}=\frac{B_{i}}{2}+\frac{1}{4}$. And finally, from 6.4 and 6.4 we get expressions for $\underline{p}_{i}$ and $\underline{p}_{j}$.

\subsection{Proof of Lemma 3.10 :}

Figure 1 shows the partition of $\operatorname{sop}\left(d F_{i}\right)$ and $\operatorname{sop}\left(d F_{j}\right)$ defined by the hypothesis. Thanks to $(I)$ we know that $\left[\bar{p}_{i}-B_{i}, \underline{p}_{i}+B_{j}\right]$ is a non-empty interval that could possibly be included in $\operatorname{sop}\left(d F_{j}\right)$.

From equation 3.2 we get,

$$
\begin{array}{ll}
F_{i}\left(p-B_{j}\right)\left(\frac{1}{2}\left(B_{j}-p\right)\right)-F_{i}\left(p+B_{i}\right) \frac{1}{2} p+p=V_{j} & \forall p \in \operatorname{sop}\left(d F_{j}\right) \\
F_{j}\left(p-B_{i}\right)\left(\frac{1}{2}\left(B_{i}-p\right)\right)-F_{j}\left(p+B_{j}\right) \frac{1}{2} p+p=V_{i} & \forall p \in \operatorname{sop}\left(d F_{i}\right)
\end{array}
$$

If $\left[\bar{p}_{i}-B_{i}, \underline{p}_{i}+B_{j}\right] \cap \operatorname{sop}\left(d F_{j}\right) \neq \varnothing$, then considering $\hat{p} \in\left[\bar{p}_{i}-B_{i}, \underline{p}_{i}+B_{j}\right] \cap \operatorname{sop}\left(d F_{j}\right)$ we have (by equation 6.6),

$$
\begin{aligned}
F_{i} \underbrace{\left(\hat{p}-B_{j}\right)}_{<\underline{p}_{i}}\left(\frac{1}{2}\left(B_{j}-\hat{p}\right)\right)-F_{i} \underbrace{\left(\hat{p}+B_{i}\right)}_{>\bar{p}_{i}} \frac{1}{2} \hat{p}+\hat{p} & =V_{j} \\
& \Longleftrightarrow \\
0\left(\frac{1}{2}\left(B_{j}-\hat{p}\right)\right)-\frac{1}{2} 1 \hat{p}+\hat{p} & =V_{j} \\
\Longleftrightarrow & \\
\frac{1}{2} \hat{p} & =V_{j} .
\end{aligned}
$$

But this would mean that we do not have a constant value for $V_{j}$, which is a contradiction. 
Therefore we have that $\left[\bar{p}_{i}-B_{i}, \underline{p}_{i}+B_{j}\right] \cap \operatorname{sop}\left(d F_{j}\right)=\varnothing$, which means that $F_{j}$ is constant on $\left[\bar{p}_{i}-B_{i}, \underline{p}_{i}+B_{j}\right]$

The methodology consists of considering $p$ in different sub intervals of $\operatorname{sop}\left(d F_{j}\right)$ which simplifies expression 6.6 and allows us to solve $F_{j}$. For $p^{*} \in\left[\underline{p}_{j}+B_{i}, \bar{p}_{i}\right]$ we have $F_{j}\left(p^{*}+B_{j}\right)=1$ since $\underline{p}_{j}+B_{i}>$ $\bar{p}_{j}-B_{j}$, and equation 6.6 becomes,

$$
\begin{aligned}
F_{j}\left(p^{*}-B_{i}\right)\left(\frac{1}{2}\left(B_{i}-p^{*}\right)\right)+\frac{1}{2} p^{*} & =V_{i} \\
\Longleftrightarrow F_{j}\left(p^{*}-B_{i}\right) & =\frac{2 V_{i}-p^{*}}{B_{i}-p^{*}} \quad \forall p^{*} \in\left[\underline{p}_{j}+B_{i}, \bar{p}_{i}\right]
\end{aligned}
$$

which means that,

$$
F_{j}(p)=\left(1-\frac{2 V_{i}-B_{i}}{p}\right) \quad \forall p \in\left[\underline{p}_{j}, \bar{p}_{i}-B_{i}\right]
$$

Similarly, for we consider $p^{*} \in\left[\underline{p}_{i}+B_{j}, \bar{p}_{j}\right]$ we have that $F_{i}\left(p^{*}+B_{i}\right)=1$ and are able to solve $F_{i}$ from equation 6.7,

$$
F_{i}(p)=\left(1-\frac{2 V_{j}-B_{j}}{p}\right) \quad \forall p \in\left[\underline{p}_{i}, \bar{p}_{j}-B_{j}\right]
$$

Now if we consider $p^{\prime} \in\left[\underline{p}_{i}, \bar{p}_{j}-B_{j}\right]$ we have that $F_{j}\left(p^{\prime}-B_{i}\right)=0$, since $\bar{p}_{j}-B_{j}<\underline{p}_{j}+B_{i}$. Therefore equation 6.7 takes on the following form,

$$
\begin{aligned}
-F_{j}\left(p^{\prime}+B_{j}\right) \frac{1}{2} p^{\prime}+p^{\prime} & =V_{i} \\
\Longleftrightarrow F_{j}\left(p^{\prime}+B_{j}\right) & =2\left(1-\frac{V_{i}}{p^{\prime}}\right) \quad \forall p^{\prime} \in\left[\underline{p}_{i}, \bar{p}_{j}-B_{j}\right]
\end{aligned}
$$

which implies that,

$$
F_{j}(p)=2\left(1-\frac{V_{i}}{p-B_{j}}\right) \quad \forall p \in\left[\underline{p}_{i}+B_{j}, \bar{p}_{j}\right]
$$

Using the same argument for $F_{i}$, if we consider $p^{\prime} \in\left[\underline{p}_{j}, \bar{p}_{i}-B_{i}\right]$ we have that $F_{i}\left(p^{\prime}-B_{j}\right)=0$ and therefore from equation 6.7 we get,

$$
F_{i}(p)=2\left(1-\frac{V_{j}}{p-B_{i}}\right) \quad \forall p \in\left[\underline{p}_{j}+B_{i}, \bar{p}_{i}\right] .
$$

With this we have completely characterized $F_{j}$ 's functional form. From before we know that $F_{j}(p)=$ $K$ for $p \in\left[\bar{p}_{i}-B_{i}, \underline{p}_{i}+B_{j}\right]$, and since $F_{j}$ must be continuous from the right, we have that $F_{j}(p)$ takes on the value of $F_{j}\left(\bar{p}_{i}-B_{i}\right)$ for all $p \in\left[\bar{p}_{i}-B_{i}, \underline{p}_{i}+B_{j}\right]$. 


$$
F_{j}(p)= \begin{cases}0 & p<\underline{p}_{j} \\ \left(1-\frac{2 V_{i}-B_{i}}{p}\right) & \underline{p}_{j} \leq p<\bar{p}_{i}-B_{i} \\ \left(1-\frac{2 V_{i}-B_{i}}{\bar{p}_{i}-B_{i}}\right) & \bar{p}_{i}-B_{i} \leq p<\underline{p}_{i}+B_{j} \\ 2\left(1-\frac{V_{i}}{p-B_{j}}\right) & \underline{p}_{i}+B_{j} \leq p<\bar{p}_{j} \\ 1 & p \geq \bar{p}_{j}\end{cases}
$$

$F_{i}$ 's partially characterized from Lemma 3.6 we know that it is continuous at $\underline{p}_{i}$,

$$
F_{i}\left(\underline{p}_{i}\right)=\left(1-\frac{2 V_{j}-B_{j}}{\underline{p}_{i}}\right)=0 \Longrightarrow \underline{p}_{i}=2 V_{j}-B_{j}
$$

To complete the proof we must find the value of $\bar{p}_{i}$ and the relationship that $V_{i}$ and $V_{j}$ must satisfy under these conditions. Let us consider $p^{*} \geq \bar{p}_{i}$, we must have the following inequality, ${ }^{12}$

$$
F_{j}\left(p^{*}-B_{i}\right)\left(\frac{1}{2}\left(B_{i}-p^{*}\right)\right)+\frac{1}{2} p^{*} \leq V_{i} .
$$

For consider $p^{*}$ sufficiently close to $\bar{p}_{i}$ such that $p^{*}-B_{i} \in\left[\bar{p}_{i}-B_{i}, \underline{p}_{i}+B_{j}\right]$, we have that $F_{j}\left(p^{*}-B_{i}\right)=K$. Thus the above inequality can be written as,

$$
\begin{aligned}
K\left(\frac{1}{2}\left(B_{i}-p^{*}\right)\right)+\frac{1}{2} p^{*} & \leq V_{i} \\
\Longleftrightarrow \frac{1}{2}(1-K) p^{*}+\frac{1}{2} K B_{i} & \leq V_{i}
\end{aligned}
$$

and since $K<1{ }^{13}$ we have that the right hand side of the above inequality is increasing in $p^{*}$. We know that for $p=\bar{p}_{i}-B_{i}$ equation 6.7 holds, therefore if we raise $i$ 's price we cannot have reduction in the firm's expected payoff. Which is a contradiction, therefore $\bar{p}_{i}=1$.

Consider $p^{\prime} \leq \underline{p}_{i}$, we must have the following inequality, ${ }^{14}$

$$
-F_{j}\left(p^{\prime}+B_{i}\right) \frac{1}{2} p^{\prime}+p^{\prime} \leq V_{i}
$$

if we consider $p^{\prime}$ sufficiently close to $\underline{p}_{i}$ so that $p^{\prime}+B_{i} \in\left[\bar{p}_{i}-B_{i}, \underline{p}_{i}+B_{j}\right]$, we have that $F_{j}\left(p+B_{i}\right)=K$. Thus the above inequality can be written as,

$$
\begin{aligned}
-K \frac{1}{2} p^{\prime}+p^{\prime} & \leq V_{i} \\
\Longleftrightarrow\left(1-\frac{K}{2}\right) p^{\prime} & \leq V_{i}
\end{aligned}
$$

\footnotetext{
${ }^{12}$ Obviously $F_{j}\left(p^{*}+B_{j}\right)=1$.

${ }^{13}$ Because $\bar{p}_{i}-B_{i}<\bar{p}_{i}$.

${ }^{14}$ Obviously $F_{j}\left(p^{\prime}-B_{i}\right)=0$.
} 
Since the right hand side is increasing in $p^{\prime}$, we shall consider in the most restrictive case $p^{\prime}=\underline{p}_{i}=$ $2 V_{j}-B_{j}$. Also, we can write $K=\left(1-\frac{2 V_{i}-B_{i}}{1-B_{i}}\right)$ from 6.8 and replacing the value of $\bar{p}_{i}$,

$$
\begin{aligned}
\left(1-\frac{1-2 V_{i}}{2\left(1-B_{i}\right)}\right)\left(2 V_{j}-B_{j}\right) & \leq V_{i} \\
\Longleftrightarrow 4 V_{i} V_{j}-2 V_{i} B_{j}+2 V_{i} B_{i}+2 V_{j}-2 V_{i}-B_{j}-4 V_{j} B_{i}+2 B_{i} B_{j} & \leq 0
\end{aligned}
$$

Another condition that must be imposed over $F_{j}$ is that it must be an increasing function. The only possible points that may infringe this condition are the points where $F_{j}$ changes its functional form, $\underline{p}_{i}+B_{j}$ and $\bar{p}_{i}$. If $p=\underline{p}_{i}+B_{j}$,

$$
\begin{aligned}
\lim _{p \rightarrow\left(\underline{p}_{i}+B_{j}\right)^{-}} F_{j}(p) & \leq F_{j}\left(\underline{p}_{i}+B_{j}\right) \\
\Longleftrightarrow 1-\frac{2 V_{i}-B_{i}}{1-B_{i}} & \leq 2\left(1-\frac{V_{i}}{\underline{p}_{i}+B_{j}-B_{j}}\right) \\
\Longleftrightarrow-4 V_{i} V_{j}+2 V_{i} B_{j}-2 V_{i} B_{i}-2 V_{j}+2 V_{i}+B_{j}+4 V_{j} B_{i}-2 B_{i} B_{j} & \leq 0
\end{aligned}
$$

Thus considering inequalities 6.9 and 6.10 we have,

$$
4 V_{i} V_{j}-2 V_{i} B_{j}+2 V_{i} B_{i}+2 V_{j}-2 V_{i}-B_{j}-4 V_{j} B_{i}+2 B_{i} B_{j}=0
$$

which completes the proof of the Lemma.

\subsection{Proof of Lemma 3.11 :}

In the proof of this Lemma we shall use similar arguments to those used in the proof of Lemma 3.10. From Figure 2 we can see the partition of $\operatorname{sop}\left(d F_{j}\right)$ and $\operatorname{sop}\left(d F_{i}\right)$ resulting from the Lemmas hypothesis. Note that the partition of $\operatorname{sop}\left(d F_{j}\right)$ does not have an interval in which $F_{j}$ is constant (like in Lemma 3.10) due to hypothesis $(I)$. For these hypothesis equations 6.6 and 6.7 still represent the equations that the firms expected payoff must satisfy.

As in the proof of Lemma 3.10, we consider different intervals of $\operatorname{sop}\left(d F_{i}\right)$ so that equation 6.7 takes on a simpler form and we can solve $F_{j}$. Let us consider $p^{*} \in\left[\underline{p}_{j}+B_{i}, \bar{p}_{i}\right]$, thanks to hypothesis $(I I)$ we have that $\underline{p}_{j}+B_{i} \geq \bar{p}_{j}-B_{j}$ which implies that $F_{j}\left(p^{*}+B_{j}\right)=1$, thus equation 6.7 becomes,

$$
\begin{aligned}
F_{j}\left(p^{*}-B_{i}\right)\left(\frac{1}{2}\left(B_{i}-p^{*}\right)\right)+\frac{1}{2} p^{*} & =V_{i} \\
F_{j}\left(p^{*}-B_{i}\right) & =\frac{2 V_{i}-p^{*}}{B_{i}-p^{*}} \quad \forall p^{*} \in\left[\underline{p}_{j}+B_{i}, \bar{p}_{i}\right]
\end{aligned}
$$


from which we can find the following expression from $F_{j}$,

$$
F_{j}(p)=\left(1-\frac{2 V_{i}-B_{i}}{p}\right) \quad \forall p \in\left[\underline{p}_{j}, \bar{p}_{i}-B_{i}\right]
$$

In the same way, we can find an expression for $F_{i}$ considering $p^{*} \in\left[\underline{p}_{i}+B_{j}, \bar{p}_{i}\right]$ and using equation 6.6 ,

$$
F_{i}(p)=\left(1-\frac{2 V_{j}-B_{j}}{p}\right) \quad \forall p \in\left[\underline{p}_{i}, \bar{p}_{j}-B_{j}\right]
$$

If $p^{\prime} \in\left[\underline{p}_{i}, \bar{p}_{j}-B_{j}\right]$ then $F_{j}\left(p^{\prime}-B_{i}\right)=0$ because $\bar{p}_{j}-B_{j} \leq \underline{p}_{j}+B_{i}$. Therefore equation 6.7 becomes,

$$
\begin{aligned}
-F_{j}\left(p^{\prime}+B_{j}\right) \frac{1}{2} p^{\prime}+p^{\prime} & =V_{i} \\
F_{j}\left(p^{\prime}+B_{j}\right) & =2\left(1-\frac{V_{i}}{p^{\prime}}\right) \quad \forall p^{\prime} \in\left[\underline{p}_{i}, \bar{p}_{j}-B_{j}\right]
\end{aligned}
$$

therefore

$$
F_{j}(p)=2\left(1-\frac{V_{i}}{p-B_{j}}\right) \quad \forall p \in\left[\underline{p}_{i}+B_{j}, \bar{p}_{j}\right]
$$

Repeating the same process with equation 6.6 we get,

$$
F_{i}(p)=2\left(1-\frac{V_{j}}{p-B_{i}}\right) \quad \forall p \in\left[\underline{p}_{j}+B_{i}, \bar{p}_{i}\right]
$$

With this we have completely characterized $F_{j}$ 's functional form,

$$
F_{j}(p)= \begin{cases}0 & p<\underline{p}_{j} \\ \left(1-\frac{2 V_{i}-B_{i}}{p}\right) & \underline{p}_{j} \leq p<\bar{p}_{i}-B_{i} \\ 2\left(1-\frac{V_{i}}{p-B_{j}}\right) & \underline{p}_{i}+B_{j} \leq p<\bar{p}_{j} \\ 1 & p \geq \bar{p}_{j}\end{cases}
$$

From Lemma 3.6 we know that $F_{i}$ is continuous at $\underline{p}_{i}$. Therefore,

$$
F_{i}\left(\underline{p}_{i}\right)=\left(1-\frac{2 V_{j}-B_{j}}{\underline{p}_{i}}\right)=0 \Longrightarrow \underline{p}_{i}=2 V_{j}-B_{j}
$$

By having an expression for $\underline{p}_{i}$, thanks to hypothesis $(I)$, we know that $\bar{p}_{i}=2 V_{j}+B_{i}$. As before, to find a relationships between $V_{i}$ and $V_{j}$ we must impose the some necessary conditions over $\underline{p}_{i}$ and $\bar{p}_{i}$. For $p^{*} \geq \bar{p}_{i}$ we must have, ${ }^{15}$

\footnotetext{
${ }^{15}$ Obviously $F_{j}\left(p^{*}+B_{j}\right)=1$.
} 


$$
F_{j}\left(p^{*}-B_{i}\right)\left(\frac{1}{2}\left(B_{i}-p^{*}\right)\right)+\frac{1}{2} p^{*} \leq V_{i}
$$

For $p^{*}$ sufficiently close to $\bar{p}_{i}$ we have an expression for $F_{j}\left(p^{*}-B_{i}\right)$, which means that the above inequality reduces,

$$
\underbrace{2\left(1-\frac{V_{i}}{p^{*}-B_{i}-B_{j}}\right)\left(B_{i}-p^{*}\right)+p^{*}}_{L\left(p^{*}\right)} \leq 2 V_{i}
$$

Notice that $L(p)$ is decreasing in $p$,

$$
\begin{aligned}
\frac{\partial L}{\partial p} & =\frac{2 V_{i}}{\left(p-B_{i}-B_{j}\right)^{2}}\left(B_{i}-p\right)-2\left(1-\frac{V_{i}}{p-B_{i}-B_{j}}\right)+1 \\
& =\frac{-2 V_{i} B_{j}}{\left(p-B_{i}-B_{j}\right)^{2}}-1 \\
& <0 .
\end{aligned}
$$

Therefore, the most restrictive case in inequality 6.12 is $p^{*}=\bar{p}_{i}$ which can be written as,

$$
\begin{aligned}
L\left(\bar{p}_{i}\right) & =2\left(1-\frac{V_{i}}{2 V_{j}-B_{j}}\right)\left(B_{i}-B_{i}-2 V_{j}\right)+2 V_{j}+B_{i} \leq 2 V_{i} \\
\Longleftrightarrow-4 V_{j}^{2}+2 V_{j} B_{j}+2 V_{i} B_{j}+2 V_{j} B_{i}-B_{i} B_{j} & \leq 0
\end{aligned}
$$

Now for $p^{\prime} \leq \underline{p}_{i}$ we must have,

$$
-F_{j}\left(p^{\prime}+B_{j}\right) \frac{1}{2} p^{\prime}+p^{\prime} \leq V_{i}
$$

and considering $p^{\prime}$ sufficiently close to $\underline{p}_{i}$ we have an expression for $F_{j}\left(p^{\prime}+B_{j}\right)$. Thus the above inequality takes on the following form,

$$
\underbrace{\left(\frac{1}{2}+\frac{2 V_{i}-B_{i}}{2\left(p^{\prime}+B_{j}\right)}\right)}_{\hat{L}\left(p^{\prime}\right)} p^{\prime} \leq V_{i}
$$

Notices that $\hat{L}(p)$ is increasing increasing in $p$, 


$$
\begin{aligned}
\frac{\partial \hat{L}}{\partial p} & =\left(\frac{1}{2}+\frac{2 V_{i}-B_{i}}{2\left(p+B_{j}\right)}\right)-p \frac{2 V_{i}-B_{i}}{2\left(p+B_{j}\right)^{2}} \\
& =\frac{1}{2}+\frac{\left(2 V_{i}-B_{i}\right)\left(p+B_{j}\right)-2 V_{i} p+B_{i} p}{2\left(p+B_{j}\right)^{2}} \\
& =\frac{1}{2}+\frac{\underline{p}_{j} B_{j}}{2\left(p+B_{j}\right)^{2}} \\
& >0
\end{aligned}
$$

Therefore the most restrictive case for inequality 6.14 is $\underline{p}_{i}=2 V_{j}-B_{j}$ which can be written as,

$$
\begin{gathered}
\hat{L}\left(\underline{p}_{i}\right)=\left(\frac{1}{2}+\frac{2 V_{i}-B_{i}}{2\left(2 V_{j}-B_{j}+B_{j}\right)}\right)\left(2 V_{j}-B_{j}\right) \leq V_{i} \\
\Longleftrightarrow 4 V_{j}^{2}-2 V_{j} B_{j}-2 V_{i} B_{j}-2 V_{j} B_{i}+B_{i} B_{j} \leq 0
\end{gathered}
$$

Hence, combining conditions 6.13 and 6.15 we get

$$
4 V_{j}^{2}-2 V_{j} B_{j}-2 V_{i} B_{j}-2 V_{j} B_{i}+B_{i} B_{j}=0
$$

which completes the proof.

\subsection{Proof of Theorem 3.12}

We separate the different cases depending on the hypothesis considered,

Case I: $\bar{p}_{i}-\underline{p}_{i}<B_{i}+B_{j}$ and $\bar{p}_{j}-\underline{p}_{j}<B_{i}+B_{j}$.

In this case both firms' strategies are characterized by Lemma 3.10, therefore we have the following system of equations,

$$
\begin{aligned}
& 4 V_{i} V_{j}-2 V_{i} B_{j}+2 V_{i} B_{i}+2 V_{j}-2 V_{i}-B_{j}-4 V_{j} B_{i}+2 B_{i} B_{j}=0 \\
& 4 V_{j} V_{i}-2 V_{j} B_{i}+2 V_{j} B_{j}+2 V_{i}-2 V_{j}-B_{i}-4 V_{i} B_{j}+2 B_{j} B_{i}=0
\end{aligned}
$$

By solving this system we get the expressions for $V_{i}$ and $V_{j}$ for this case.

Case II: $\bar{p}_{i}-\underline{p}_{i}<B_{i}+B_{j}$ and $\bar{p}_{j}-\underline{p}_{j}=B_{i}+B_{j}$.

In this case, firm $j$ 's strategy is characterized by Lemma 3.10 and firm $i$ 's strategy is characterized by Lemma 3.11, therefore we have the following system of equations, ${ }^{16}$

$$
4 V_{i} V_{j}-2 V_{i} B_{j}+2 V_{i} B_{i}+2 V_{j}-2 V_{i}-B_{j}-4 V_{j} B_{i}+2 B_{i} B_{j}=0
$$

\footnotetext{
${ }^{16}$ The case when we have $\bar{p}_{j}-\underline{p}_{j}<B_{i}+B_{j}$ and $\bar{p}_{i}-\underline{p}_{i}=B_{i}+B_{j}$, we must simply interchange $i$ with $j$ in the system of equations.
} 


$$
4 V_{i}^{2}-2 V_{i} B_{i}-2 V_{j} B_{i}-2 V_{i} B_{j}+B_{j} B_{i}=0
$$

By solving this system we get the expressions for $V_{i}$ and $V_{j}$ for this case.

Case III: $\bar{p}_{i}-\underline{p}_{i}=B_{i}+B_{j}$ and $\bar{p}_{j}-\underline{p}_{j}=B_{i}+B_{j}$.

In this case both firms' strategies are characterized by Lemma 3.11, therefore we have the following system of equations,

$$
\begin{aligned}
& 4 V_{j}^{2}-2 V_{j} B_{j}-2 V_{i} B_{j}-2 V_{j} B_{i}+B_{i} B_{j}=0 \\
& 4 V_{i}^{2}-2 V_{i} B_{i}-2 V_{j} B_{i}-2 V_{i} B_{j}+B_{j} B_{i}=0
\end{aligned}
$$

By solving this system we get the expressions for $V_{i}$ and $V_{j}$ for this case.

\subsection{Proof of Theorem 3.14:}

We consider two different cases,

a) $\bar{p}_{j}-\underline{p}_{j}<2\left(B_{i}+B_{j}\right)$

b) $\bar{p}_{j}-\underline{p}_{j} \geq 2\left(B_{i}+B_{j}\right)$

First, let us analyze case $a$ ). Given the hypothesis of the theorem we can see the partition of the interval $\left[\underline{p}_{j}, \bar{p}_{j}\right]$ in Figure 3 and as before we will derive firm $j$ 's strategy using equation 3.2,

$$
F_{j}\left(p-B_{i}\right)\left(\frac{1}{2}\left(B_{i}-p\right)\right)-F_{j}\left(p+B_{j}\right) \frac{1}{2} p+p=V_{i} \quad \forall p \in \operatorname{sop}\left(d F_{i}\right)
$$

For $p^{\prime}>\bar{p}_{j}-B_{j}$, we have that $F_{j}\left(p+B_{j}\right)=1$ therefore the above equation becomes,

$$
F_{j}\left(p^{\prime}-B_{i}\right)\left(\frac{1}{2}\left(B_{i}-p^{\prime}\right)\right)-\frac{1}{2} p^{\prime}+p^{\prime}=V_{i} \quad \forall p \in\left[\bar{p}_{j}-B_{j}, \bar{p}_{i}\right]
$$

where we can solve for the following expression of $F_{j}{ }^{17}$

$$
F_{j}(p)=\left(1-\frac{2 V_{i}-B_{i}}{p}\right) \quad \forall p \in\left[\bar{p}_{j}-B_{i}-B_{j}, \bar{p}_{i}-B_{i}\right]
$$

For $p^{*}<\underline{p}_{j}+B_{i}$ we have that $F_{j}\left(p^{*}-B_{i}\right)=0$ and therefore from equation 3.2 we get

$$
-F_{j}\left(p+B_{j}\right) \frac{1}{2} p+p=V_{i}
$$

\footnotetext{
${ }^{17}$ It is possible that $\bar{p}_{j}-B_{i}-B_{j}<\underline{p} j$ in which case the expression for $F_{j}$ would be for a smaller interval.
} 
which implies, ${ }^{18}$

$$
F_{j}(p)=2\left(1-\frac{V_{i}}{p-B_{j}}\right) \quad \forall p \in\left[\underline{p}_{i}+B_{j}, \underline{p}_{j}+B_{i}+B_{j}\right]
$$

Since $\bar{p}_{j}-\underline{p}_{j}<2\left(B_{i}+B_{j}\right)$, we have $\bar{p}_{j}-B_{i}-B_{j}<\underline{p}_{j}+B_{i}+B_{j}$. Therefore, we have two completely different expressions for $F_{j}$ in an interval where the domains in 6.16 and 6.17 intersect which is a contradiction. ${ }^{19}$

Now we analyze case $b$ ). Given the hypothesis of the theorem, the partition of the interval $\left[\underline{p}_{j}, \bar{p}_{j}\right]$ can be seen in Figure 3, but with a larger distance between $\underline{p}_{j}$ and $\bar{p}_{j}$.

For $p^{\prime}>B_{i}$, and since cumulative distribution functions are increasing, from 3.2 we have,

$$
\begin{gathered}
F_{j}\left(p^{\prime}-B_{i}\right) \underbrace{\left(\frac{1}{2}\left(B_{i}-p^{\prime}\right)\right)}_{<0, \text { since } p^{\prime}>B_{i}}-F_{j}\left(p^{\prime}+B_{j}\right) \frac{1}{2} p^{\prime}+p^{\prime}=V_{i} \\
\Longleftrightarrow F_{j}\left(p^{\prime}+B_{j}\right)\left(\frac{1}{2}\left(B_{i}-p^{\prime}\right)\right)-F_{j}\left(p^{\prime}+B_{j}\right) \frac{1}{2} p^{\prime}+p^{\prime} \leq V_{i}
\end{gathered}
$$

rewriting the last inequality,

$$
F_{j}\left(p^{\prime}+B_{j}\right)\left(\frac{B_{i}}{2}-p^{\prime}\right)+p^{\prime} \leq V_{i}
$$

If we consider $p^{\prime}$ so that $p^{\prime}+B_{j} \in\left[\bar{p}_{j}-B_{i}-B_{j}, \bar{p}_{i}-B_{i}\right]$, this implies that $p^{\prime}+B_{j}>B_{i}+B_{j}$ because

$$
\begin{aligned}
\bar{p}_{j}-\underline{p}_{j} & \geq 2\left(B_{i}+B_{j}\right) \\
\bar{p}_{j}-B_{i}-B_{j} & \geq \underbrace{\underline{p} j}_{>0}+B_{i}+B_{j} \\
\bar{p}_{j}-B_{i}-B_{j} & >B_{i}+B_{j}
\end{aligned}
$$

Therefore, we can write equation 6.18 replacing the expression of $F_{j}\left(p^{\prime}+B_{j}\right)$ from 6.16 giving us the following inequality,

$$
\begin{aligned}
\left(1-\frac{2 V_{i}-B_{i}}{p^{\prime}+B_{j}}\right)\left(\frac{B_{i}}{2}-p^{\prime}\right)+p^{\prime} & \leq V_{i} \\
\Longleftrightarrow p^{\prime} & \leq\left(B_{i}+B_{j}\right)
\end{aligned}
$$

\footnotetext{
${ }^{18}$ Note that these arguments can also be used to find expressions for $F_{j}(p)$ when $p \in\left[\underline{p}_{i}+B_{j}, \underline{p}_{i}+B_{i}+B_{j}\right] \cup\left[\bar{p}_{j}-B_{i}-B_{j}, \bar{p}_{i}-B_{i}\right]$ for case $b$.

${ }^{19}$ Remember that hypothesis $I$ assures us that $\underline{p}_{i}+B_{j}<\bar{p}_{i}-B_{i}$.
} 
The most restrictive case for $p^{\prime}$ we have that

$$
\bar{p}_{i}-B_{i}-B_{j} \leq B_{i}+B_{j}
$$

or equivalently,

$$
\bar{p}_{i}-\underbrace{\underline{p}_{i}}_{>0}<2\left(B_{i}+B_{j}\right) .
$$

which we know from case $a$ ) reversing the roles of $i$ and $j$ that cannot be an equilibrium.

\subsection{Proof of Lemma 3.6 part II :}

Now we prove that $F_{j}$ is continuous in $\underline{p}_{j}$ when $B_{i}=0$ and firms are in a single sided poaching equilibrium, ${ }^{20}$ that means that $\bar{p}_{i}-\underline{p}_{j}>B_{i}$ and $\bar{p}_{j}-\underline{p}_{i}<B_{j} \cdot{ }^{21}$. Let us assume that $F_{j}$ is discontinuous in $\underline{p}_{j}$

$$
F_{j}\left(\underline{p}_{j}\right)=a \geq 0
$$

We have the following expressions for the firms expected payoffs,

$$
\begin{gathered}
-F_{i}(p) \frac{1}{2} p+p=V_{j} \\
-F_{j}(p) \frac{1}{2} p+\frac{1}{2} p=V_{i}
\end{gathered}
$$

As in Theorem 3.8, if we consider $p^{\prime}>\bar{p}_{i}$ then from the first equality we would have $\frac{1}{2} p=V_{j}$, which cannot be since $V_{j}$ is constant for all $p \in \operatorname{sop}\left(d F_{j}\right)$, hence $\bar{p}_{i} \geq \bar{p}_{j}$. Now if we consider $p^{\prime}<\underline{p}_{j}$, then from the second equality we have that $\frac{1}{2} p^{\prime}=V_{i}$ which cannot be since $V_{i}$ is constant for all $p \in \operatorname{sop}\left(d F_{i}\right)$, hence $\underline{p}_{j} \leq \underline{p}_{i}$

Since $B_{j}>0$, we know that $F_{i}$ is continuous in $\underline{p}_{i}$ and that that $F_{i}(p)$ is constant for values of $p<\underline{p}_{j}$, therefore for $F_{i}$ to be continuous in $\underline{p}_{i}$ we must have that constant equal to zero (i.e $\underline{p}_{i} \leq \underline{p}_{j}$ ), hence

$$
\underline{p}_{i}=\underline{p}_{j}
$$

As before, we can find the functional form of $F_{i}$ and $F_{j}$ using the expected payoff equations ${ }^{22}$,

\footnotetext{
${ }^{20}$ Note that we have interchanged the roles of $i$ and $j$ for the proof of this part of the Lemma so the reader may follow the proof more easily since the arguments are quite similar to those used in the proof of Theorem 3.8.

${ }^{21}$ For these hypothesis do not have to analyze the case in which $B_{j}=0$.

${ }^{22}$ Note that we do not need continuity in $F_{i}$ to find these expressions. See proof of Theorem 3.8 for details
} 


$$
F_{j}(p)= \begin{cases}0 & p<\underline{p}_{j} \\ \left(1-\frac{2 V_{i}}{p}\right) & \underline{p}_{j} \leq p<\bar{p}_{i} \\ \left(1-\frac{2 V_{i}}{\bar{p}_{i}}\right) & \bar{p}_{i} \leq p<\bar{p}_{j} \\ 1 & p \geq \bar{p}_{j}\end{cases}
$$

and

$$
F_{i}(p)= \begin{cases}0 & p<\underline{p}_{i} \\ 2\left(1-\frac{V_{j}}{p}\right) & \underline{p}_{j} \leq p<\bar{p}_{i} \\ 1 & p \geq \bar{p}_{i}\end{cases}
$$

Giving an expression for $F_{j}$, we can derive the following equality for $F_{j}\left(\underline{p}_{j}\right)$,

$$
\begin{gathered}
F_{j}\left(\underline{p}_{j}\right)=a \\
\Longleftrightarrow \\
1-\frac{2 V_{i}}{\underline{p}_{j}}=a \\
\Longleftrightarrow \\
\underline{p}_{j}=\frac{2 V_{i}}{1-a}
\end{gathered}
$$

and using continuity of $F_{i}$ in $\underline{p}_{i}$, we have that $\underline{p}_{i}=V_{j}$. Note that if we consider $p^{\prime}<\underline{p}_{i}$ must have a reduction firm $i$ expected payoff. Therefore, from 3.2 we have,

$$
\begin{aligned}
\underbrace{-F_{j}\left(p^{\prime}\right) \frac{1}{2} p^{\prime}}_{=0, \text { since } \underline{p}_{i}=\underline{p}_{j}}+\frac{1}{2} p^{\prime} & \leq V_{i} \\
\Longleftrightarrow \frac{1}{2} p^{\prime} & \leq V_{i}
\end{aligned}
$$

since we have that the left hand side is increasing in $p^{\prime}$, we must make the bound hold in the most restrictive case, $p^{\prime}=\underline{p}_{i}=\underline{p}_{j}$ therefore we have,

$$
\begin{aligned}
\frac{2 V_{i}}{1-a} & \leq 2 V_{i} \\
\Longleftrightarrow & \leq 0
\end{aligned}
$$

therefore $a=0$, completing this part of the proof. 


\subsection{Proof of Lemma 3.6 part III:}

Now we prove that $F_{i}$ is continuous in $\underline{p}_{i}$ when $B_{j}=0$ and firms are in a double sided poaching equilibrium. We only have two cases to analyze,

1. Double Poaching Equilibrium: Case II

2. Double Poaching Equilibrium: Case III

since the double poaching equilibrium described by case I does not occur when one bond is equal to zero (see Figure 5).

We will characterize the equilibrium considering a possible discontinuity in $\underline{p}_{i}$ (i.e. $F_{i}\left(\underline{p}_{i}\right)=a>0$ ) and then prove that $V_{i}$ is decreasing in $a$, which will allow us to argue that $i$ does not have incentives to have a discontinuity in his strategy proving this part of the Lemma.

Double Poaching Equilibrium: Case II. In this case, we have that $\bar{p}_{i}-\underline{p}_{i}<B_{i}$ and $\bar{p}_{j}-\underline{p}_{j}=B_{i}$. Since $B_{i}>0$, from Lemma 3.11 we have the characterizations of $i$ 's strategy and the following equation for the firms expected payoff, ${ }^{23}$

$$
2 V_{i}^{2}-V_{i} B_{i}-V_{j} B_{i}=0
$$

Now we must derive a result similar to that of Lemma 3.10 for $j$, but without assuming continuity of $F_{i}$ in $\underline{p}_{i}$. As before, we can find $F_{j}$ 's functional form using equation 3.2 in the same way we did for the proof of Lemma $3.10^{24}$. Thus the firms' strategies take on the following form,

$$
\begin{aligned}
& F_{j}(p)= \begin{cases}0 & p<\underline{p}_{j} \\
\left(1-\frac{2 V_{i}-B_{i}}{p}\right) & \underline{p}_{j} \leq p<\bar{p}_{i}-B_{i} \\
\left(1-\frac{2 V_{i}-B_{i}}{\bar{p}_{i}-B_{i}}\right) & \bar{p}_{i}-B_{i} \leq p<\underline{p}_{i} \\
2\left(1-\frac{V_{i}}{p}\right) & \underline{p}_{i} \leq p<\bar{p}_{j} \\
1 & p \geq \bar{p}_{j}\end{cases} \\
& F_{i}(p)= \begin{cases}0 & p<\underline{p}_{i} \\
\left(1-\frac{2 V_{j}}{p}\right) & \underline{p}_{i} \leq p<\bar{p}_{j} \\
2\left(1-\frac{V_{j}}{p-B_{i}}\right) & \underline{p} j+B_{i} \leq p<\bar{p}_{i} \\
1 & p \geq \bar{p}_{i}\end{cases}
\end{aligned}
$$

\footnotetext{
${ }^{23}$ Note that in the proof of Lemma 3.11 we do not need continuity of $F_{j}$ in $\underline{p}_{j}$.

${ }^{24}$ In deriving the $F_{j}$ 's functional form it is not necessary to have $B_{j}>0$.
} 
With an expression for $F_{i}$ we can derive the following equality for $F_{i}\left(\underline{p}_{i}\right)$,

$$
\begin{aligned}
F_{i}\left(\underline{p}_{i}\right) & =a \\
\Longleftrightarrow 1-\frac{2 V_{j}}{\underline{p}_{i}} & =a \\
\Longleftrightarrow \underline{p}_{i} & =\frac{2 V_{j}}{1-a}
\end{aligned}
$$

If we consider $p^{*} \geq \bar{p}_{i}$ we have a reduction in $i$ 's expected payoff,

$$
F_{j}\left(p^{*}-B_{i}\right)\left(\frac{1}{2}\left(B_{i}-p^{*}\right)\right)+\frac{1}{2} p^{*} \leq V_{i}
$$

If we consider $p^{*}$ sufficiently close to $\bar{p}_{i}$ such that $p^{*}-B_{i} \in\left[\bar{p}_{i}-B_{i}, \underline{p}_{i}\right]$, we have that $F_{j}\left(p^{*}-B_{i}\right)=K$. Thus the above inequality can be written as,

$$
\begin{aligned}
K\left(\frac{1}{2}\left(B_{i}-p^{*}\right)\right)+\frac{1}{2} p^{*} & \leq V_{i} \\
\Longleftrightarrow \frac{1}{2}(1-K) p^{*}+\frac{1}{2} K B_{i} & \leq V_{i}
\end{aligned}
$$

which cannot be because the left hand side is an increasing function in $p^{*}$, therefore $\bar{p}_{i}=1$. Now if we consider $p^{\prime}<\underline{p}_{i}$ we have the following inequality,

$$
-F_{j}\left(p^{\prime}\right) \frac{1}{2} p^{\prime}+p^{\prime} \leq V_{i}
$$

considering $p^{\prime}$ sufficiently close to $\underline{p}_{i}$ so that $p^{\prime}+B_{i} \in\left[\bar{p}_{i}-B_{i}, \underline{p}_{i}+B_{j}\right]$, we have that $F_{j}\left(p^{\prime}\right)=K$ and the above inequality may be written as,

$$
\begin{aligned}
-K \frac{1}{2} p^{\prime}+p^{\prime} & \leq V_{i} \\
\Longleftrightarrow\left(1-\frac{c}{2}\right) p^{\prime} & \leq V_{i}
\end{aligned}
$$

The right hand side of the above inequality is increasing in $p^{\prime}$ we shall consider the most restrictive case $p^{\prime}=\underline{p}_{i}=\frac{2 V_{j}}{1-a}$ and express $K$ in terms of the expression $F_{j}$,

$$
\begin{aligned}
\left(1-\frac{1-2 V_{i}}{2\left(1-B_{i}\right)}\right) \frac{2 V_{j}}{1-a} & \leq V_{i} \\
\Longleftrightarrow 2 V_{i} V_{j}-2 V_{j} B_{i}+V_{j}-V_{i}+V_{i} B_{i}+V_{i} a-V_{i} B_{i} a & \leq 0
\end{aligned}
$$


We must also impose that $F_{j}$ be an increasing function. This means that in $\underline{p}_{i}$ we must have the following inequality,

$$
\begin{aligned}
\lim _{p \rightarrow\left(\underline{p}_{i}\right)^{-}} F_{j}(p) & \leq F_{j}\left(\underline{p}_{i}\right) \\
\Longleftrightarrow 1-\frac{2 V_{i}-B_{i}}{1-B_{i}} & \leq 2\left(1-\frac{V_{i}(1-a)}{2 V_{j}}\right) \\
\Longleftrightarrow-2 V_{i} V_{j}+2 V_{j} B_{i}-V_{j}+V_{i}-V_{i} B_{i}-V_{i} a+V_{i} B_{i} a & \leq 0
\end{aligned}
$$

combining inequalities 6.20 and 6.21 with equation 6.19 , we have that $V_{i}$ and $V_{j}$ must satisfy the following system of equations,

$$
\begin{gathered}
2 V_{i}^{2}-V_{i} B_{i}-V_{j} B_{i}=0 \\
2 V_{i} V_{j}-2 V_{j} B_{i}+V_{j}-V_{i}+V_{i} B_{i}+V_{i} a-V_{i} B_{i} a=0
\end{gathered}
$$

which give us,

$$
V_{i}=\frac{1}{4}\left[3 B_{i}-1+\sqrt{\left(1-B_{i}\right)\left(1+3 B_{i}-4 B_{i} a\right)}\right]
$$

From this last expression of $V_{i}$ it can be seen that $i$ 's expected payoff is decreasing in $a$. Therefore, $i$ does not have incentives to have a discontinuity in his strategy at $\underline{p}_{i}$, proving the Lemma for this case.

Double Poaching Equilibrium: Case III. In this case we have that $\bar{p}_{i}-\underline{p}_{i}=B_{i}$ and $\bar{p}_{j}-\underline{p}_{j}=B_{i}$. Since $B_{i}>0$ from Lemma 3.11 we have the characterizations of $i$ 's strategy and, as in Case II, the firms expected payoff satisfy equation 6.19 .

Now we must derive a result similar to that of Lemma 3.11 for $j$, but without assuming continuity of $F_{i}$ in $\underline{p}_{i}$. As before, we can find $F_{j}$ 's functional form equation 3.2 in the same way we did for the proof of Lemma $3.10^{25}$. Thus the firms' strategies take on the following form,

$$
F_{j}(p)= \begin{cases}0 & p<\underline{p}_{j} \\ \left(1-\frac{2 V_{i}-B_{i}}{p}\right) & \underline{p}_{j} \leq p<\bar{p}_{i}-B_{i} \\ 2\left(1-\frac{V_{i}}{p}\right) & \underline{p}_{i} \leq p<\bar{p}_{j} \\ 1 & p \geq \bar{p}_{j}\end{cases}
$$

\footnotetext{
${ }^{25}$ In deriving the $F_{j}$ 's functional form it is not necessary to have $B_{j}>0$.
} 


$$
F_{i}(p)= \begin{cases}0 & p<\underline{p}_{i} \\ \left(1-\frac{2 V_{j}}{p}\right) & \underline{p}_{i} \leq p<\bar{p}_{j} \\ 2\left(1-\frac{V_{j}}{p-B_{i}}\right) & \underline{p}_{j}+B_{i} \leq p<\bar{p}_{i} \\ 1 & p \geq \bar{p}_{i}\end{cases}
$$

With an expression for $F_{i}$, we can derive the following equality for $F_{i}\left(\underline{p}_{i}\right)$,

$$
\begin{aligned}
F_{i}\left(\underline{p}_{i}\right) & =a \\
\Longleftrightarrow 1-\frac{2 V_{j}}{\underline{p}_{i}} & =a \\
\Longleftrightarrow \underline{p}_{i} & =\frac{2 V_{j}}{1-a}
\end{aligned}
$$

which also means that $\bar{p}_{i}=B_{i}+\frac{2 V_{j}}{1-a}$

If we consider $p^{*} \geq \bar{p}_{i}$ we have a reduction in $i$ 's expected payoff,

$$
F_{j}\left(p^{*}-B_{i}\right)\left(\frac{1}{2}\left(B_{i}-p^{*}\right)\right)+\frac{1}{2} p^{*} \leq V_{i}
$$

Considering $p^{*}$ sufficiently close to $\bar{p}_{i}$ we can replacing the expression for $F_{j}\left(p^{*}-B_{i}\right)$,

$$
\begin{aligned}
2\left(1-\frac{V_{i}}{p^{*}-B_{i}}\right)\left(\frac{1}{2}\left(B_{i}-p^{*}\right)\right)+\frac{1}{2} p^{*} & \leq V_{i} \\
\Longleftrightarrow B_{i}-\frac{1}{2} p^{*} & \leq 0
\end{aligned}
$$

The left hand side of the above inequality is decreasing in $p^{*}$, therefore evaluating in the most restrictive case $\left(p^{\prime}=\bar{p}_{i}=B_{i}+\frac{2 V_{j}}{1-a}\right)$ we have,

$$
B_{i}-\frac{2 V_{j}}{1-a} \leq 0
$$

If we consider $p^{\prime}<\underline{p}_{i}$, again we have a reduction in $i$ 's expected payoff,

$$
-F_{j}\left(p^{\prime}\right) \frac{1}{2} p^{\prime}+p^{\prime} \leq V_{i}
$$

and if we consider $p^{\prime}$ sufficiently close to $\underline{p}_{i}$ we can replace the expression for $F_{j}(p)$,

$$
\begin{aligned}
-\frac{1}{2}\left(1-\frac{2 V_{i}-B_{i}}{p^{\prime}}\right) p^{\prime}+p^{\prime} & \leq V_{i} \\
\Longleftrightarrow p^{\prime}-B_{i} & \leq 0
\end{aligned}
$$


The right hand side of the above inequality is increasing in $p^{\prime}$, therefore evaluating in the most restrictive case $p^{\prime}=\underline{p}_{i}=\frac{2 V_{j}}{1-a}$ we have,

$$
\frac{2 V_{j}}{1-a}-B_{i} \leq 0
$$

putting together inequalities 6.22 and 6.23 with equation 6.19 , we have that $V_{i}$ and $V_{j}$ must satisfy the following system of equations,

$$
\begin{gathered}
2 V_{i}^{2}-V_{i} B_{i}-V_{j} B_{i}=0 \\
\frac{2 V_{j}}{1-a}-B_{i}=0
\end{gathered}
$$

and solving this system of equations, we get the following value for $V_{i}$,

$$
V_{i}=\frac{1}{4}\left[B_{i}+B_{i} \sqrt{5-4 a}\right]
$$

From this last expression of $V_{i}$ it can be seen that $i$ 's expected payoff is decreasing in $a$. Therefore, $i$ does not have incentives to have a discontinuity is his strategy at $\underline{p}_{i}$, completing the proof of the Lemma. 


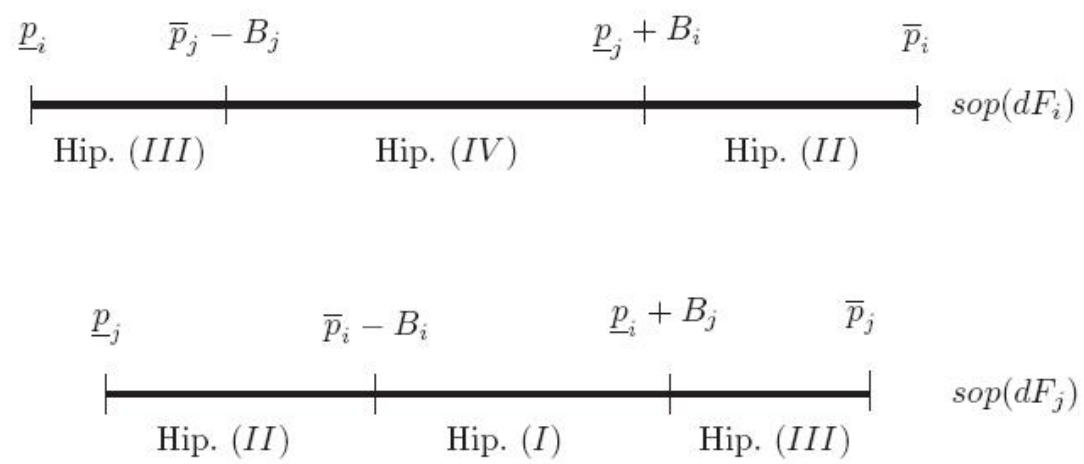

Figure 1: Partition of $d F_{i}$ and $d F_{j}$ for Lemma 3.10
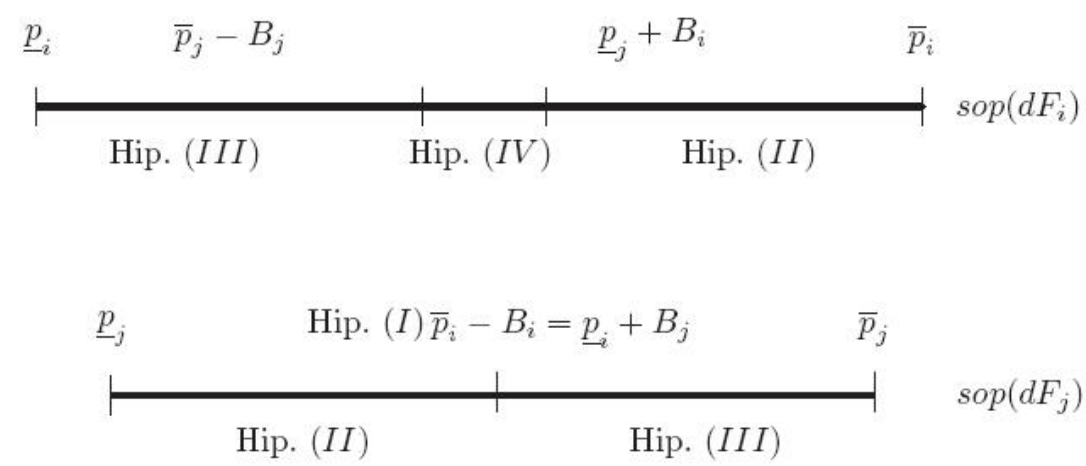

Figure 2: Partition of $d F_{i}$ and $d F_{j}$ for Lemma 3.11

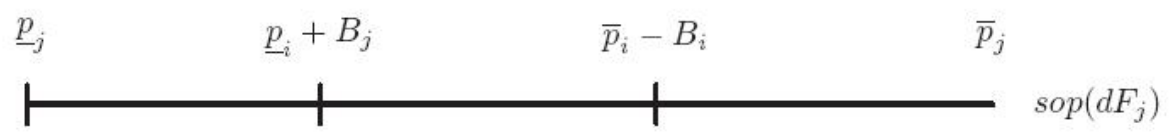

Hip. ( $($ )

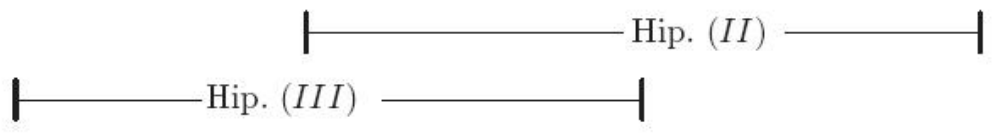

Figure 3: Partition of $d F_{i}$ and $d F_{j}$ for Theorem 3.14 

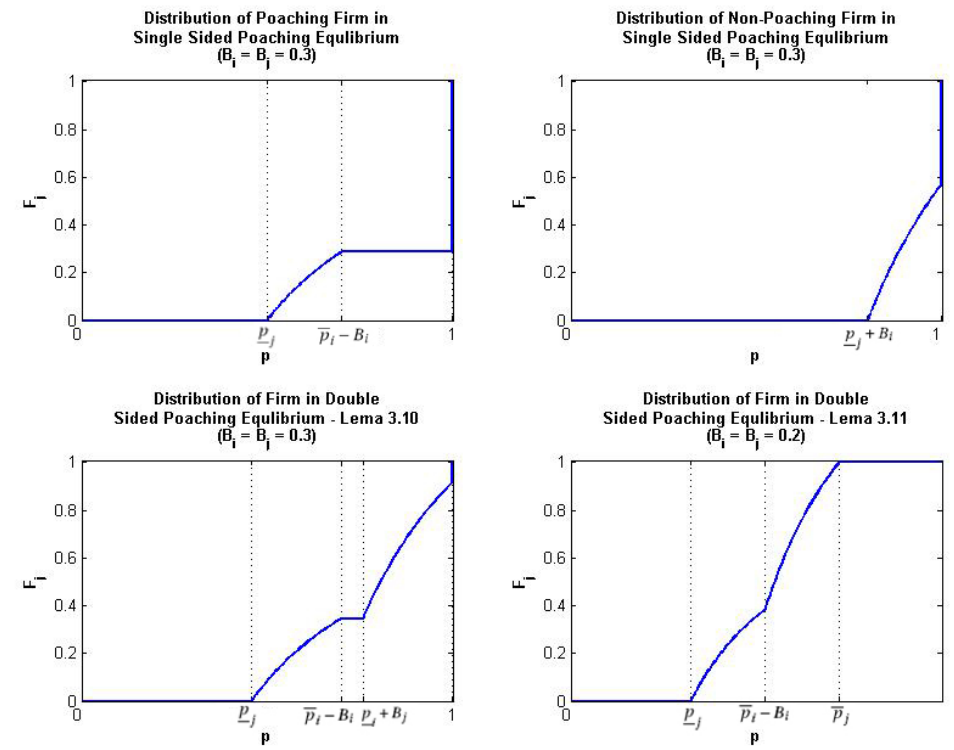

Figure 4: Example of Firms Mixed Strategy
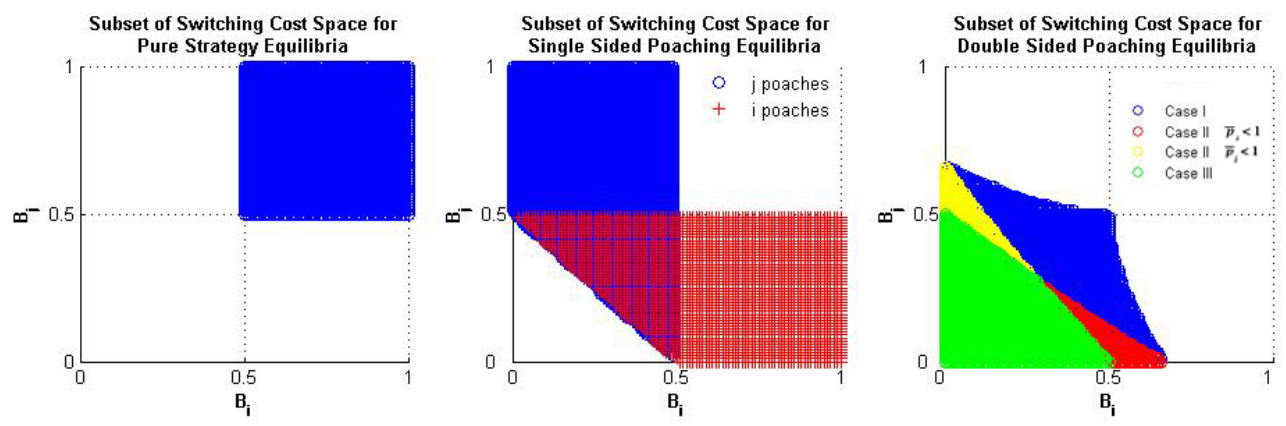

Figure 5: Subset of Space $\left(B_{i}, B_{j}\right)$ for Different Equilibria
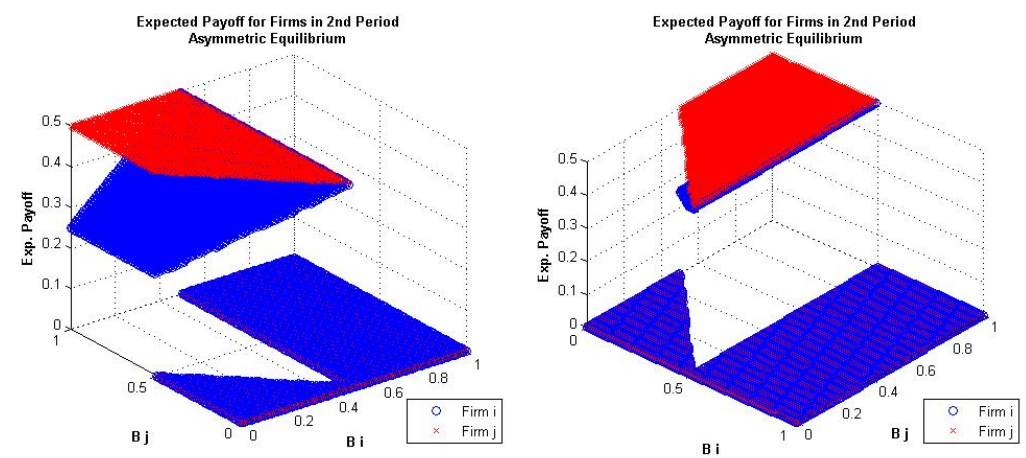

Figure 6: Single Sided Poaching Equilibrium 

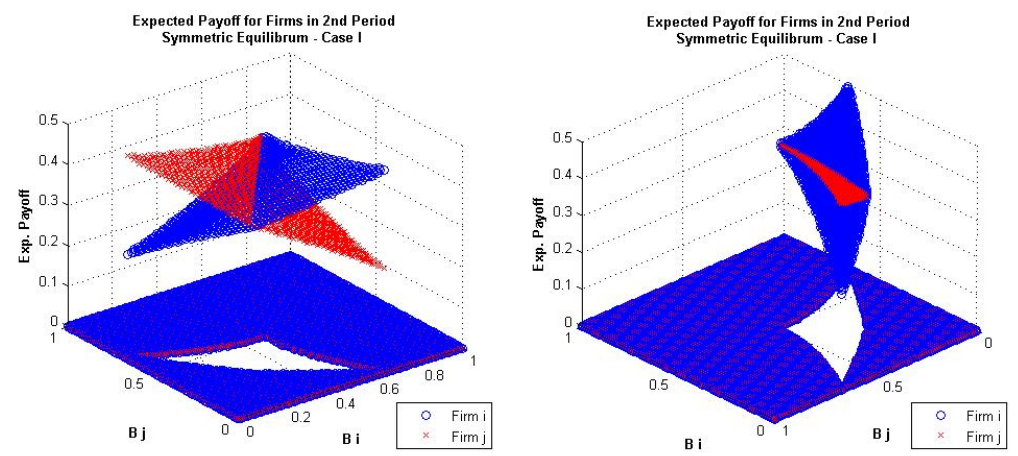

Figure 7: Double Sided Poaching Equilibrium - Case I
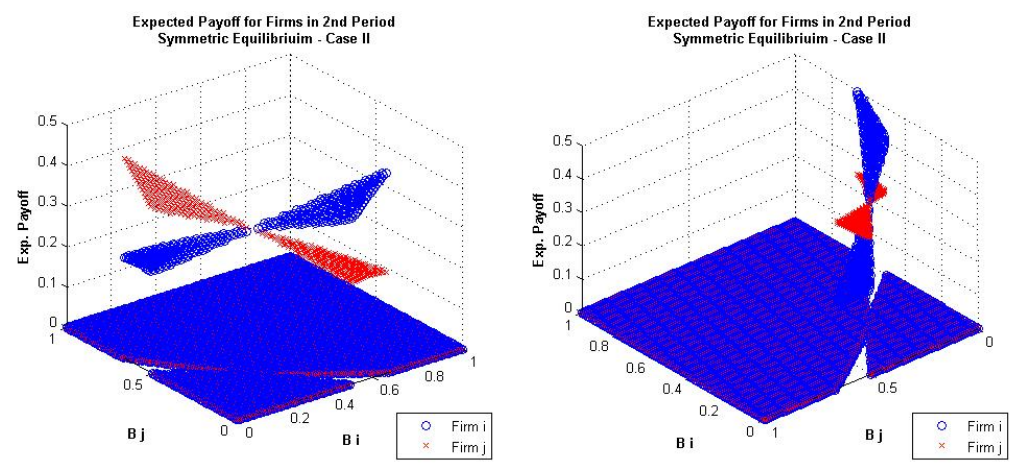

Figure 8: Double Sided Poaching Equilibrium - Case II
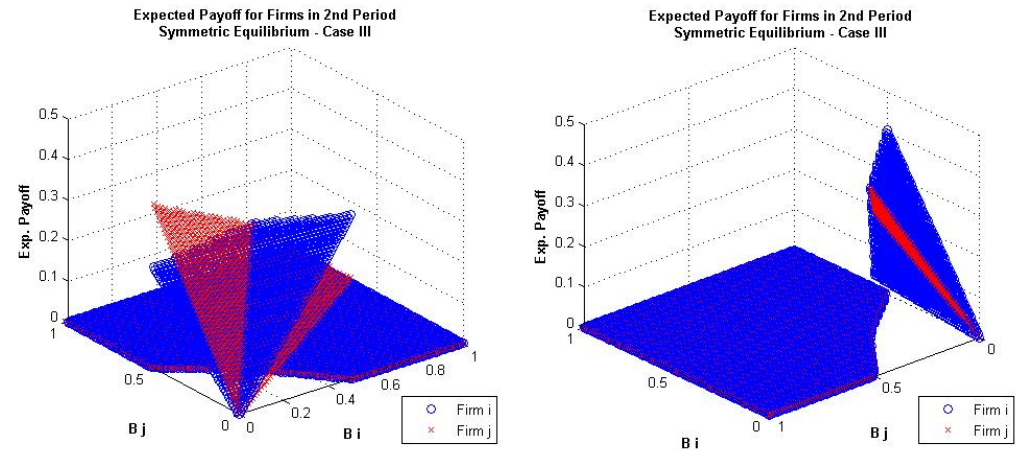

Figure 9: Double Sided Poaching Equilibrium - Case III 


\section{References}

[1] BEGGS, A. and KLEMPERER, P.D. (1992), "Multi-Period Competition with Switching Costs", Econometrica, Vol. 60, No. 3, (May, 1992) 651-666.

[2] CAMINAL, R. and MATUTES, C. (1990) "Endogenous Switching Costs in a Duoply Model”, International Journal of Industrial Organization, 8: 353-374.

[3] CHEN, Y. (1997) "Paying Customers to Switch", Journal of Economics and Management Strategy, 6: 877-897.

[4] DASGUPTA, P. y MASKIN, E. (1986) "The Existence of Equilibrium in Discontinous Economic Games, I: Theory” The Review of Economic Studies Vol. 53, No. 1, 1-26.

[5] EDGEWORTH, F. (1925) “The pure theory of monopoly" Papers Relating to Political Economy Vol. 1, pp. 111-42.

[6] FARRELL, J. and SHAPIRO, C. (1988), "Dynamic Competition with Switching Costs”, The RAND Journal of Economics Vol. 19, No. 1 (Spring, 1988), 123-137.

[7] FARRELL J. and KLEMPERER, P.D. (2001), "Coordination and Lock-in: Competition with Switching Costs and Network Effects”, Preliminary Draft.

[8] GALETOVIC, A. (2004), "Microeconimía II" Departamento de Ingenieria Industrial, Facultad de Ciencias Físicas y Matemáticas, Universidad de Chile.

[9] HOLT, C (1994), "Oligopoly price Competition with Incomplete Information: Convergence to MixedStrategy Equilibria” Working Paper.

[10] KLEMPERER, P.D (1987a), "Markets with Consumer Switching Costs", Quarterly Journal of Economics, 102: 375-394.

[11] KLEMPERER, P.D. (1987b), “The Competitivness of Markets with Switching Costs", Rand Journal of Economics, 18: 138-150.

[12] KLEMPERER, P.D. (1995), "Competition when Consumers have Switching Costs: An Overview with Applications to Industrial Organization, Macroeconomics, and International Trade", The Review of Econometric Studies, Vol. 62, No. 4, 515-539.

[13] PADILLA, A. J. (1992), "Mixed Pricing in Oligopoly with Consumer Switching Costs", International Journal of Industrial Organization, 10: 393-412.

[14] SALANIÉ, B. (200) “Microeconomics of Market Failures”, Massachusetts Institute of Technology 2000.

[15] SHILONY, Y. (1977) “Mixed Pricing in Oligopoly”, Journal of Economic Theory, 14: 373-388.

[16] SONNENSCHEIN, H. (1968) "The Dual of Duopoly is Complementary Monopoly: Or, Two of Cournot's Theories are One", Journal of Political Economy, 76: 316-18. 
[17] STAAHL, T. and VAGSTAD, S. (2000), "Consumer Heterogeneity and Pricing in a Duopoly with Consumer Switching Costs”, Econometric Society World Congress 2000 Contributed Papers, 0449, Econometric Society.

[18] VARIAN, H. (1980) "A Model of Sales”, The American Economic Review Vol. 70, No. 4 (Sep. 1980), 651659. 